\title{
On the calculation of vibrational energy relaxation rate constants from centroid molecular dynamics simulations
}

\author{
Qiang Shi and Eitan Geva \\ Department of Chemistry, University of Michigan, Ann Arbor, Michigan 48109-1055
}

(Received 11 June 2003; accepted 4 August 2003)

\begin{abstract}
We explore the use of centroid molecular dynamics (CMD) for calculating vibrational energy relaxation (VER) rate constants of high-frequency molecular vibrations in the condensed phase. We employ our recently proposed linear-response-theory-based approach to VER [Q. Shi and E. Geva, J. Chem. Phys. 118, 7562 (2003)], to obtain a new expression for the VER rate constant in terms of a correlation function that can be directly obtained from CMD simulations. We show that the new expression reduces to a centroid Landau-Teller-type formula in the golden-rule regime. Unlike previously proposed CMD-based approaches to VER, the new formula does not involve additional assumptions beyond the inherent CMD approximation. The new formula has the same form as the classical Landau-Teller formula, and quantum effects enter it in two ways: (1) The initial sampling and subsequent dynamics are governed by the centroid potential, rather than the classical potential; (2) The classical force is replaced by the corresponding centroid symbol. The application of the new method is reported for three model systems: (1) A vibrational mode coupled to a harmonic bath, with the coupling exponential in the bath coordinates; (2) A diatomic molecule coupled to a short linear chain of Helium atoms; (3) A "breathing sphere" diatomic molecule in a two-dimensional monoatomic Lennard-Jones liquid. It is confirmed that CMD is able to capture the main features of the force-force correlation function rather well, in both time and frequency domains. However, we also find that $\mathrm{CMD}$ is unable to accurately predict the high-frequency tail of the quantum-mechanical power spectrum of this correlation function, which limits its usefulness for calculating VER rate constants of high-frequency molecular vibrations. The predictions of CMD are compared with those obtained via the linearized-semiclassical initial-value-representation (LSC-IVR) method, which does yield accurate predictions of high-frequency VER rate constants. The reasons underlying these observations are discussed in terms of the similarities and differences between these two approaches. (C) 2003 American Institute of Physics. [DOI: 10.1063/1.1613636]
\end{abstract}

\section{INTRODUCTION}

The problem of vibrational energy relaxation (VER) in the condensed phase has received much attention over the last few decades. ${ }^{1-39}$ The VER rate provides a sensitive probe of intramolecular dynamics and solute-solvent interactions, which are known to have a crucial impact on other important properties, such as chemical reactivity, solvation dynamics, and transport coefficients. The calculation of VER rate constants has presented theoretical chemistry with an ongoing challenge due to the high frequency of most molecular vibrations (in the sense that $\hbar \omega / k_{B} T \gg 1$ ). One implication of the high frequency is that VER is often found to be slow, due to the low density of accepting modes with matching frequencies, and therefore cannot be obtained directly from nonequilibrium MD simulations. This problem is usually circumvented by resorting to the Landau-Teller (LT) formula, which gives the VER rate constant in terms of the Fourier transform (FT), at the vibrational frequency, of a certain short-lived force-force correlation function (FFCF), which can be calculated from equilibrium MD simulations with a rigid solute. It should be noted that the derivation of the LT formula is based on several assumptions, namely: weak coupling between the solute and solvent, separation of time scales (such that the VER lifetime is much longer than the correlation time of the FFCF), and the rotating wave approximation (RWA) ${ }^{40}$

Another difficulty has to do with the fact that extracting the very small high-frequency Fourier components of the FFCF can become extremely difficult due to statistical noise accompanying all real-life simulations. This difficulty is often dealt with by using an extrapolation of the exponential gap law, which usually emerges at low frequencies, to much higher frequencies. ${ }^{41,42}$ An alternative, yet similar, approach combines a short time expansion of the FFCF with a parameterized ansatz that exhibits an exponential gap law behavior at high frequencies, and whose FT can be calculated analytically. ${ }^{43-51}$

Yet another fundamental difficulty imposed by the fact that $\hbar \omega / k_{B} T \gg 1$, is that the quantum-mechanical $\mathrm{FFCF}$, rather than the classical FFCF, should be used in the LT formula. The exact calculation of real-time quantummechanical correlation functions for general many-body systems remains far beyond the reach of currently available computer resources, due to the exponential scaling of the computational effort with the number of degrees of freedom (DOF) ${ }^{52}$ The most popular approach for dealing with this difficulty is to first evaluate the FT of the classical FFCF, and 
then multiply the result by a frequency-dependent quantum correction factor $(Q C F) .{ }^{1,53-68}$ Various approximate QCFs have been proposed in the literature. Unfortunately, estimates obtained from different QCFs can differ by orders of magnitude, and particularly so when high-frequency vibrations are involved. Thus, finding more rigorous ways for computing VER rate constants is clearly highly desirable.

Several strategies have been proposed in order to address the challenge of providing an effective, computationally feasible, and versatile approximate method for calculating quantum-mechanical real-time correlation functions. These methods are based on various approaches, including a mixed quantum-classical treatment, ${ }^{69-74}$ analytical continuation, ${ }^{46,75-81}$ centroid molecular dynamics (CMD), ${ }^{82-106}$ quantum mode coupling theory, ${ }^{107-109}$ and the semiclassical (SC) approximation. ${ }^{52,110-128}$ These methods have been applied, with relative success, to a rather extensive set of systems and processes. However, the application of these methods to VER in condensed-phase systems has been rather limited. ${ }^{46,51,82,103,104}$ It should be noted that such applications are desirable for two main reasons: (1) They may give rise to better ways for calculating VER rate constants, as well as understanding its underlying quantum-dynamical mechanism; (2) The highly quantum-mechanical nature of VER, which often leads to deviations by orders of magnitude between the classical predictions and experimental results, provides an excellent platform for testing and comparing different methods for their ability to capture quantum effects in condensed phase systems.

To this end, we have recently considered the application of the linearized semiclassical initial-value-representation (LSC-IVR) method of Miller et al. ${ }^{10,119,125,129-132}$ for calculating the quantum-mechanical FFCF. ${ }^{133}$ In this case, the quantum-mechanical FFCF is approximated by a classicallike expression, where Wigner transforms replace the corresponding classical quantities and the dynamics is fully classical. In practice, the computation of the multidimensional Wigner integrals was based on a local-harmonicapproximation (LHA), and the method was therefore designated LHA-LSC-IVR. The LHA-LSC-IVR method has been applied to several nontrivial model systems and its predictions were found to be in good agreement with other estimates or experiment. These studies have also shown that the VER of high-frequency molecular vibrations is dominated by purely quantum-mechanical fluctuations of the force, which are unaccounted for in calculations based on classical MD simulations.

In the present paper, we consider the application of another approach, which is based on the CMD method, for calculating quantum-mechanical VER rate constants in condensed phase systems. Previous attempts by Voth and co-workers ${ }^{82}$ and Poulsen and co-workers ${ }^{46,103,104}$ to use CMD for this purpose, were complicated by the fact that the application of CMD is restricted to correlation function where at least one of the operators is linear in the coordinates and/or momenta (see Sec. II). This is definitely not the case in most relevant VER problems, where the force in the above mentioned FFCF involves a highly nonlinear function of the coordinates. To overcome this difficulty, these workers proposed to augment CMD with additional approximations. In the present paper, we show that this is not necessary, and that one can derive an approximate expression for the VER rate constant which is only based on the CMD approximation, and avoids any other additional approximations. To this end, we employ our recently proposed linear-response-based theory of VER, ${ }^{40}$ which enables us to express the VER rate constant in terms of a correlation function that CMD can be directly applied to. By imposing on the resulting expression for the VER rate constant the same assumptions that lead to the LT formula, ${ }^{40}$ we then derive a centroid LT-like expression for the VER rate constant, which can be calculated directly from CMD simulations. It should be emphasized that the derivation of this centroid LT formula does not involve any additional approximation beyond the original CMD approximation $^{90}$ (see Sec. III). It therefore allows for a clean test of the applicability of the CMD approximation to VER, without mixing CMD with additional, essentially uncontrolled, approximations.

The structure of the remainder of this paper is as follows. An overview of the relevant CMD theory is given in Sec. II. The derivation of the centroid LT formula is given in Sec. III. The predictions of the centroid LT formula are reported for several model systems in Sec. IV. Finally, a general discussion on the applicability of CMD to VER, and its relationship to the LHA-LSC-IVR-based studies of VER, is given in Sec. V.

\section{AN OVERVIEW OF CENTROID MOLECULAR DYNAMICS (CMD)}

In its most recent formulation, ${ }^{89,90}$ centroid dynamics has been shown to be based on the following phase-space operator (given here in 1D, for simplicity),

$$
\hat{\phi}\left(x_{c}, p_{c}\right)=\frac{\hbar}{2 \pi} \int_{-\infty}^{\infty} d \xi \int_{-\infty}^{\infty} d \eta e^{i \xi\left(\hat{x}-x_{c}\right)+i \eta\left(\hat{p}-p_{c}\right)-\beta \hat{H}},
$$

where $x_{c}$ and $p_{c}$ are the centroid position and momentum, respectively, and $\beta=1 / k_{B} T$ is the inverse temperature. A central role is reserved for the trace of this operator, which corresponds to the centroid density,

$$
\rho_{c}\left(x_{c}, p_{c}\right)=\operatorname{Tr}\left[\hat{\phi}\left(x_{c}, p_{c}\right)\right] .
$$

The centroid approach also associates a classical-like centroid symbol, $A_{c}\left(x_{c}, p_{c}\right)$, with each quantum dynamical observable, $A(\hat{x}, \hat{p})$, which is defined by

$$
A_{c}\left(x_{c}, p_{c}\right)=\operatorname{Tr}\left[\hat{\phi}\left(x_{c}, p_{c}\right) A(\hat{x}, \hat{p})\right] / \rho_{c}\left(x_{c}, p_{c}\right) .
$$

The centroid density, $\rho_{c}\left(x_{c}, p_{c}\right)$, turns out to have a classical-like form, which is similar to that of the classical Boltzmann distribution,

$$
\rho_{c}\left(x_{c}, p_{c}\right)=e^{-\beta p_{c}^{2} / 2 m} \rho_{c}\left(x_{c}\right) \equiv e^{-\beta p_{c}^{2} / 2 m} e^{-\beta V_{c m}\left(x_{c}\right)} .
$$

$V_{c m}\left(x_{c}\right)=-\ln \left[\rho_{c}\left(x_{c}\right)\right] / \beta$ in Eq. (4) is called the centroid potential. It is distinctly different from the classical potential and can be written in terms of a constrained imaginary-time path integral, 
TABLE I. The centroid formulation of quantum statistical mechanics.

$$
\begin{aligned}
& \begin{array}{lcl}
\hline \hline Z= & \text { Standard } & \text { Centroid } \\
\hline \operatorname{Tr}\left(e^{-\beta \hat{H}}\right) & \iint \frac{d x_{c} d p_{c}}{2 \pi \hbar} e^{-\beta\left[p_{c}^{2} / 2 m+V_{c m}\left(x_{c}\right)\right]}
\end{array} \\
& \langle\hat{A}\rangle=\quad \operatorname{Tr}\left(e^{-\beta \hat{H}} \hat{A}\right) / Z \quad \quad \iint \frac{d x_{c} d p_{c}}{2 \pi \hbar} e^{-\beta\left[p_{c}^{2} / 2 m+V_{c m}\left(x_{c}\right)\right]} A_{c}\left(x_{c}, p_{c}\right) / Z \\
& C_{\mathrm{BA}}^{\mathrm{Kubo}}(t)=\quad \int_{0}^{\beta} \frac{d \lambda}{\beta} \operatorname{Tr}\left(e^{-\beta \hat{H}} \hat{B} \hat{A}(t+i \lambda / \hbar)\right) \quad \iint \frac{d x_{c} d p_{c}}{2 \pi \hbar} e^{-\beta\left[p_{c}^{2} / 2 m+V_{c m}\left(x_{c}\right)\right]} B_{c}\left(x_{c}, p_{c}\right) A_{c}\left[x_{c}, p_{c} ; t\right] \\
& e^{-\beta V_{c m}\left(x_{c}\right)} \equiv \rho_{c}\left(x_{c}\right)=\left(\frac{2 \pi \beta \hbar^{2}}{m}\right)^{1 / 2} \int_{x(0)=x(\beta \hbar)} D x(\lambda) \delta\left[x_{c}-(\beta \hbar)^{-1} \int_{0}^{\beta \hbar} d \lambda x(\lambda)\right] \exp \{-\mathcal{S}[x(\lambda)] / \hbar\} \\
& =\lim _{P \rightarrow \infty}\left(\frac{2 \pi \beta \hbar^{2}}{m}\right)^{1 / 2}\left(\frac{m P}{2 \pi \beta \hbar^{2}}\right)^{P / 2} \int d x_{1} \cdots \int d x_{P} \delta\left(x_{c}-\frac{1}{P} \sum_{k=1}^{P} x_{k}\right) \exp \left\{-\mathcal{S}\left[x_{1}, \ldots, x_{P}\right] / \hbar\right\},
\end{aligned}
$$

with

$$
\begin{aligned}
\frac{1}{\hbar} \mathcal{S}[x(\lambda)] & =\lim _{P \rightarrow \infty} \frac{1}{\hbar} \mathcal{S}\left[x_{1}, \ldots, x_{P}\right] \\
& =\frac{1}{\hbar} \int_{0}^{\beta \hbar} d \lambda\left[\frac{1}{2} m[\dot{x}(\lambda)]^{2}+V[x(\lambda)]\right]
\end{aligned}
$$

and

$$
\begin{aligned}
\frac{1}{\hbar} \mathcal{S}\left[x_{1}, \ldots, x_{P}\right]= & \beta\left\{\sum_{k=1}^{P} \frac{m P}{2 \beta^{2} \hbar^{2}}\left(x_{k}-x_{k+1}\right)^{2}\right. \\
& \left.+\frac{1}{P} \sum_{k=1}^{P} V\left(x_{k}\right)\right\} .
\end{aligned}
$$

In Eq. (7), $x_{P+1}=x_{1}$. It should be noted that $\rho_{c}\left(x_{c}\right)$ is proportional to the probability density of finding a classical cyclic chain polymer consisting of $P$ beads, which are connected by harmonic springs and subject to the potential $V(x) / P$, with their center of mass (the centroid) at $x=x_{c}$. The centroid also corresponds to the zero-frequency normal mode of the chain polymer. The imaginary-time path integral in Eq. (5) can be computed using classical molecular dynamics or Monte Carlo simulations (PIMD and PIMC, respectively) for relatively complex many-body systems. ${ }^{134,135}$

The above definitions form the basis for an exact classical-like formulation of quantum statistical mechanics, which is summarized in Table I. The last line in Table I is of particular importance since it relates the classical-like twotime centroid correlation function with the exact Kubotransformed quantum-mechanical correlation function, when $\hat{B}$ is proportional to $\hat{x}$ (or $\hat{p}$ ),

$$
\begin{aligned}
& \frac{1}{2 \pi \hbar} \int d x_{c} \int d p_{c} e^{-\beta\left[p_{c}^{2} / 2 m+V_{c m}\left(x_{c}\right)\right]} x_{c} A_{c}\left[x_{c}, p_{c} ; t\right] \\
& =\frac{1}{\beta} \int_{0}^{\beta} d \lambda \operatorname{Tr}\left(e^{-\beta \hat{H}} \hat{x} \hat{A}(t+i \hbar \lambda)\right) .
\end{aligned}
$$

It should be noted that Kubo-transformed correlation functions can be related to the corresponding regular correlation functions via a well known identity. ${ }^{91}$ However, the relationship in Eq. (8) is of little practical use since the exact time dependence of the centroid symbol $A_{c}\left[x_{c}, p_{c} ; t\right]$ is given by

$$
A_{c}\left(x_{c}, p_{c} ; t\right)=\operatorname{Tr}\left[e^{-i \hat{H} t / \hbar} \hat{\phi}_{c}\left(x_{c}, p_{c}\right) e^{i \hat{H} t / \hbar} \hat{A}\right] / \rho_{c}\left(x_{c}, p_{c}\right),
$$

and requires the same amount of effort to calculate as in standard quantum mechanics. The CMD method is based on circumventing this difficulty by imposing the following CMD approximation: ${ }^{90}$

$$
e^{-i \hat{H} t / \hbar} \hat{\phi}_{c}\left(x_{c}, p_{c}\right) e^{i \hat{H} t / \hbar} \approx \hat{\phi}_{c}\left[x_{c}(t), p_{c}(t)\right],
$$

such that

$$
A_{c}\left[x_{c}, p_{c} ; t\right] \approx A_{c}\left[x_{c}(t), p_{c}(t)\right] .
$$

Here $x_{c}(t)$ and $p_{c}(t)$ are propagated as classical-like position and momentum variables on the centroid potential, $V_{c m}\left(x_{c}\right)$ [cf. Eqs. (4) and (5)].

We also note for later use that a centroid correlation function similar to that in Eq. (8), except that $x_{c}$ is replaced by $x_{c}^{n}$, where $n$ is a positive integer, can be shown to be identical to the corresponding high order Kubo-transformed correlation function. ${ }^{91}$ For example,

$$
\begin{aligned}
\frac{1}{2 \pi \hbar} \int d x_{c} \int d p_{c} e^{-\beta\left[p_{c}^{2} / 2 m+V_{c m}\left(x_{c}\right)\right]} x_{c}^{2} A_{c}\left[x_{c}, p_{c} ; t\right] \\
=\frac{2}{\beta^{2}} \int_{0}^{\beta} d \beta_{1} \int_{0}^{\beta_{1}} d \beta_{2} \operatorname{Tr}\left(e^{-\beta \hat{H}} \hat{x}\left(-i \beta_{1} / \hbar\right)\right. \\
\left.\quad \times \hat{x}\left(-i \beta_{2} / \hbar\right) \hat{A}(t)\right) .
\end{aligned}
$$

The CMD approximation for the correlation function in Eq. (12) can then be obtained by applying Eq. (11) to $A_{c}\left[x_{c}, p_{c} ; t\right]$. 
TABLE II. Commonly used quantum correction factors.

\begin{tabular}{cccc}
\hline \hline Name & $A_{Q}(\omega)$ & Assumption & Refs. \\
\hline Standard & $2 /\left(1+e^{-\beta \hbar \omega}\right)$ & $\operatorname{Re}[C(t)] \approx C^{\mathrm{Cl}}(t)$ & $1,62,64$ \\
Harmonic & $\beta \hbar \omega /\left(1-e^{-\beta \hbar \omega}\right)$ & $\begin{array}{c}\text { One-phonon process } \\
\text { in harmonic bath }\end{array}$ & $54,61-63$ \\
Schofield & $e^{\beta \hbar \omega / 2}$ & $C(t) \approx C^{\mathrm{Cl}}(t+i \hbar \beta / 2)$ & 66 \\
Egelstaff & $\frac{e^{\beta \hbar \omega / 2}}{\widetilde{C}^{\mathrm{Cl}}(\omega)} \int_{-\infty}^{\infty} d t e^{i \omega t} C^{\mathrm{Cl}}\left(\sqrt{t^{2}+(\beta \hbar / 2)^{2}}\right)$ & $C(t) \approx C^{\mathrm{Cl}}(\sqrt{t(t+i \beta \hbar / 2)})$ & $44,53,62,67,68$ \\
Harmonic/Schofield & $\sqrt{A_{Q}^{\text {harmonic }}(\omega) A_{Q}^{\text {Schofield }}(\omega)}$ & $\begin{array}{c}\text { Multiphonon process } \\
\text { in harmonic bath }\end{array}$ & $55,57,60$ \\
\hline \hline
\end{tabular}

\section{THE CENTROID LANDAU-TELLER FORMULA}

\section{A. The standard Landau-Teller formula}

We consider the following general quantum mechanical Hamiltonian of a harmonic vibrational mode coupled to a bath;

$$
\hat{H}=\hat{H}_{s}+\hat{H}_{b}+\hat{H}_{b s} \equiv \hat{H}^{0}+\hat{H}_{b s},
$$

where

$$
\hat{H}_{s}=\frac{\hat{p}^{2}}{2 \mu}+\frac{1}{2} \mu \omega^{2} \hat{q}^{2}
$$

is the Hamiltonian of the vibrational mode under investigation $(\hat{q}, \hat{p}, \mu$, and $\omega$ are the corresponding coordinate, momentum, reduced mass, and frequency, respectively),

$$
\hat{H}_{b}=\sum_{i=1}^{N} \frac{\left(\hat{P}^{(i)}\right)^{2}}{2 M^{(i)}}+\hat{V}\left(\hat{Q}^{(1)}, \ldots, \hat{Q}^{(N)}\right)
$$

is the Hamiltonian of the bath, which consists of the other intermolecular and intramolecular degrees of freedom $\left(\left\{\hat{Q}^{(i)}\right\}=\hat{\mathbf{Q}},\left\{\hat{P}^{(i)}\right\}=\hat{\mathbf{P}},\left\{M^{(i)}\right\}\right.$ and $\hat{V}\left(\hat{Q}^{(1)}, \ldots, \hat{Q}^{(N)}\right)=V(\hat{\mathbf{Q}})$ are the corresponding coordinates, momenta, masses, and potential energy, respectively), and

$$
\hat{H}_{b s}=\alpha \hat{q} \hat{F}\left(\hat{Q}^{(1)}, \ldots, \hat{Q}^{(N)}\right)=\alpha \hat{q} \hat{F}(\hat{\mathbf{Q}})
$$

is the system-bath coupling term, where $\alpha$ is the systembath coupling parameter (to be later used in order to keep track of the order of the perturbation in the golden rule limit). The system-bath coupling term, $\hat{H}_{b s}$, is assumed to be linearized in the vibrational coordinate, $\hat{q}$. We also assume that the force on the vibrational mode, $\hat{F}(\hat{\mathbf{Q}})$, is a function of the bath coordinates only. It should be noted that this type of potential force is typically a highly nonlinear function of the bath coordinates, and is usually found to make a larger contribution to the VER rate constant than momenta-dependent centrifugal forces.

The standard description of VER is based on the assumption that it follows simple rate kinetics, ${ }^{43,54,136}$

$$
\frac{d}{d t}\left\langle\delta \hat{H}_{s}\right\rangle=-\frac{1}{T_{1}}\left\langle\delta \hat{H}_{s}\right\rangle,
$$

where $\delta \hat{H}_{s}=\hat{H}_{s}-\left\langle\hat{H}_{s}\right\rangle_{0}$, and $\left\langle\hat{H}_{s}\right\rangle_{0}=\hbar \omega / 2+\hbar \omega /\left(e^{\beta \hbar \omega}\right.$ -1 ) is the vibrational energy at thermal equilibrium (at the weak coupling limit). The central quantity in Eq. (17) is the VER rate constant, $1 / T_{1}$, which is given by the LandauTeller (LT) formula, ${ }^{9,43,137-148}$

$$
\frac{1}{T_{1}}=\frac{1-e^{-\beta \hbar \omega}}{\beta \hbar \omega} \frac{\beta}{2 \mu} \widetilde{C}(\omega) .
$$

Here

$$
\widetilde{C}(\omega)=\int_{-\infty}^{\infty} d \tau e^{i \omega \tau} C(\tau)
$$

is the FT of the free bath FFCF,

$$
C(\tau)=\left\langle\delta \hat{F}_{0}(\tau) \delta \hat{F}\right\rangle_{0},
$$

where $\langle\hat{A}\rangle_{0}=\operatorname{Tr}\left[e^{-\beta \hat{H}_{b}} \hat{A}\right] / \operatorname{Tr}\left[e^{-\beta \hat{H}_{b}}\right], \delta \hat{F}=\hat{F}-\langle\hat{F}\rangle_{0}$, and

$$
\delta \hat{F}_{0}(t)=e^{i \hat{H}_{b} t / \hbar} \delta \hat{F} e^{-i \hat{H}_{b} t / \hbar} .
$$

Thus, Eq. (18) puts the VER rate constant in terms of the FT, at the vibrational frequency, of the quantum-mechanical FFCF, which is evaluated with the vibrational mode frozen at its equilibrium position $(\hat{q}=0)$.

It should be noted that the derivation of Eqs. (17) and (18) is based on the Bloch-Redfield theory (BRT), ${ }^{43,137-146}$ and relies on the validity of the following assumptions: (1) Weak system-bath coupling, to the extent that first-order time-dependent perturbation theory (Fermi's golden rule) applies; (2) Separation of time scales, such that the VER lifetime is much longer than the correlation time of the FFCF; (3) The rotating wave approximation ( $R W A)$, which amounts to the removal of rapidly oscillating terms, and results in the decoupling of population relaxation from phase relaxation.

It is important to note that the LT formula, Eq. (18), involves the quantum FFCF, rather than the classical FFCF. The most popular approach for dealing with this difficulty is to first evaluate the FT of the classical FFCF, and then multiply the result by a frequency-dependent quantum correction factor $(Q C F), A_{Q}(\omega):^{1,53-68}$

$$
\widetilde{C}(\omega) \approx A_{Q}(\omega) \widetilde{C}^{\mathrm{Cl}}(\omega) .
$$

Here $\quad \widetilde{C}^{\mathrm{Cl}}(\omega)=\int_{-\infty}^{\infty} d t e^{i \omega t} C^{\mathrm{Cl}}(t), \quad$ where $\quad C^{\mathrm{Cl}}(t)$ $=\left\langle\delta F_{0}(t) \delta F\right\rangle_{0}^{\mathrm{Cl}}$ is the classical FFCF $\left[\langle\cdots\rangle_{0}^{\mathrm{Cl}}\right.$ corresponds to averaging over the classical Boltzmann phase space distribution, and the time evolution of $\delta F_{0}(t)$ is governed by the classical free bath Hamiltonian]. Table II lists some of the more popular QCFs. However, estimates provided by the 
various QCFs can differ by orders of magnitude, and particularly so when high-frequency vibrations are involved. ${ }^{55,57,65}$ For example, Egorov et al. have recently estimated $1 / T_{1}$ for $\mathrm{O}_{2}$ in liquid $\mathrm{O}_{2}$, at $70 \mathrm{~K}$, and found the following spread of values that were based on different QCFs: $0.00095 \mathrm{~s}^{-1}$ (standard), $0.015 \mathrm{~s}^{-1}$ (harmonic), $270 \mathrm{~s}^{-1}$ (Egelstaff), $4030 \mathrm{~s}^{-1}$ (Schofield). (The experimental value under these conditions is $1 / T_{1}=360 \mathrm{~s}^{-1}$.) Similar lack of uniqueness has also been observed in other systems. ${ }^{59,65}$

In this paper, we consider using CMD in order to approximate the quantum mechanical VER rate constant. As discussed in Sec. II, using CMD to directly calculate a correlation function requires that at least one of the operators is linear in the coordinates and/or momenta. Unfortunately, the LT formula involves the autocorrelation function of the force operator, which is typically a highly nonlinear function of the bath coordinates. Hence, it appears that the FFCF cannot be calculated directly from CMD simulations. Previous attempts to overcome this problem were all based on introducing one or more of the following additional approximations: ${ }^{46,82,84,86,88,91,103,104}$ (1) Approximate analytic continuation; (2) A second-order cumulant approximation; (3) Approximate semiclassical representation of nonlinear operators; (4) Approximate classical representation of nonlinear operators. All of these approximations have serious drawbacks. For example, the cumulant approximation will fail if the dynamics is not Gaussian, and will not lead to the correct classical limit. In a recent paper, Reichman et al. have derived a formal relationship between nonlinear centroid time correlation functions and high-order Kubo transformed quantum ones [cf. Eq. (12)]. ${ }^{91}$ However, in practice, a numerically exact transformation of these high-order Kubo-transformed correlation functions into standard ones, becomes very expensive, and therefore impractical in the case of highly nonlinear and/or many-body operators.

\section{B. Linear-response theory of VER}

The above discussion suggests that the LT formula cannot be evaluated directly from CMD simulations without the help of additional approximations. In this section, we consider an alternative strategy which is based on expressing the VER rate constant in terms of another correlation function that can be calculated directly from CMD simulations. To this end, we will derive the VER rate constant from linearresponse theory (LRT), ${ }^{149}$ rather than from BRT.

We start out by considering the possibility of using standard LRT. The analysis in this case follows closely that in Ref. 40, and starts by assuming that the initial state of the overall system has the following from:

$$
\hat{\rho}(0)=\frac{e^{-\beta(\hat{H}+f \hat{A})}}{\operatorname{Tr}\left[e^{-\beta(\hat{H}+f \hat{A})}\right]} .
$$

Here $\hat{H}$ is the actual Hamiltonian of the overall system [cf. Eq. (13)], $f$ is a coupling parameter, and $\hat{A}$ is a perturbation, which will be kept unspecified for the time being. We next assume that the perturbation, $f \hat{A}$, is small enough, such that $\hat{\rho}(0)$ can be substituted by its expansion to first order in $f$;

$$
\hat{\rho}(0) \approx \frac{e^{-\beta \hat{H}}}{Z}\left[1-f \int_{0}^{\beta} d \lambda \delta \hat{A}(-i \hbar \lambda)\right],
$$

where $\quad Z=\operatorname{Tr}\left(e^{-\beta \hat{H}}\right), \quad \delta \hat{A}=\hat{A}-\langle\hat{A}\rangle_{\mathrm{eq}}, \quad\langle\hat{A}\rangle_{\mathrm{eq}}$ $=\operatorname{Tr}\left(e^{-\beta \hat{H}} \hat{A}\right) / Z$, and $\hat{A}(-i \hbar \lambda)=e^{\lambda H} \hat{A} e^{-\lambda H}$. A measurement of VER is initiated by turning the perturbation off at $t=0$, and monitoring the expectation value of the vibrational energy as a function of time, as it progresses toward its equilibrium value. For $\hat{\rho}(0)$ in Eq. (24) this yields

$$
\begin{aligned}
\left\langle\delta \hat{H}_{s}\right\rangle(t) & \equiv \operatorname{Tr}\left[\hat{\rho}(0) \delta \hat{H}_{s}(t)\right] \\
& =-f \int_{0}^{\beta} d \lambda\left\langle\delta \hat{A}(-i \hbar \lambda) \delta \hat{H}_{s}(t)\right\rangle_{\mathrm{eq}},
\end{aligned}
$$

where $\delta \hat{H}_{s}(t)=e^{i \hat{H} t / \hbar} \delta \hat{H}_{s} e^{-i \hat{H} t / \hbar}$.

Our basic hypothesis is that VER follows rate kinetics, and can therefore be characterized by a rate constant, $k$;

$$
\frac{\left\langle\delta \hat{H}_{s}\right\rangle(t)}{\left\langle\delta \hat{H}_{s}\right\rangle(0)}=e^{-k t} .
$$

The VER rate constant, $k$, can then be written in the following way:

$$
-\frac{\left\langle\delta \dot{\hat{H}}_{s}\right\rangle(t)}{\left\langle\delta \hat{H}_{s}\right\rangle(0)}=k e^{-k t}=k \quad \text { at } t \ll k^{-1} .
$$

Substituting Eq. (25) into Eq. (27), and using the explicit expression for the vibrational energy flux,

$$
\dot{\hat{H}}_{s}=\frac{i}{\hbar}\left[\hat{H}, \hat{H}_{s}\right]=-\frac{\alpha}{\mu} \hat{p} \hat{F}(\hat{\mathbf{Q}}),
$$

then leads to the following general expression for the VER rate constant:

$$
k=\frac{\alpha}{\mu} \frac{\int_{0}^{\beta} d \lambda\langle\delta \hat{A}(-i \hbar \lambda)[\hat{p} \hat{F}](t)\rangle_{\mathrm{eq}}}{\int_{0}^{\beta} d \lambda\left\langle\delta \hat{A}(-i \hbar \lambda) \delta \hat{H}_{s}\right\rangle_{\mathrm{eq}}} .
$$

Equation (29) allows us to express the VER rate constant in terms of a Kubo-transformed correlation function, $\int_{0}^{\beta} d \lambda\langle\delta \hat{A}(-i \hbar \lambda)[\hat{p} \hat{F}](t)\rangle_{\text {eq }}$, which is distinctly different from the FFCF. Furthermore, the fact that the perturbation $\hat{A}$ is still unspecified, implies that Eq. (29) actually represents a whole class of new expressions for the VER rate constant, which correspond to different choices of $\hat{A}$. One particular choice, namely $\hat{A}=\hat{q}$, leads to a correlation function which can be directly obtained from CMD simulations. Unfortunately, we have found that this particular choice would lead to non-LT VER. ${ }^{40}$ More specifically, we have shown in Ref. 40 that when $\hat{A}=\hat{q}$, first order time-dependent perturbation theory (equivalent to Fermi's golden rule) cannot account for VER. Thus, in this case VER is dominated by non-LT higher order terms in the perturbation expansion, and its rate is given in terms of multiple-time force correlation functions. 
Non-LT VER is interesting for its own sake and will be explored in future work. However, in this paper, we are primarily interested in finding ways to calculate the rate constant in the case of LT-type VER. In Ref. 40, we have shown that substituting a perturbation of the form $\hat{A}=(\delta \hat{q})^{2}$, can give rise to an expression that reduces to the LT formula upon imposing the conditions under which BRT is valid (weak system-bath coupling, separation of time scales and RWA). Unfortunately, the corresponding Kubo-transformed correlation function, $\int_{0}^{\beta} d \lambda\left\langle\delta(\delta \hat{q})^{2}(-i \hbar \lambda)[\hat{p} \hat{F}](t)\right\rangle_{\mathrm{eq}}$, cannot be obtained directly from CMD simulations. The origin of this problem and its resolution in terms of an extended version of LRT are discussed in the following section.

\section{Extended linear response theory of VER}

The source of the dilemma encountered in the previous section can be traced back to our choice of a Bolzmanized initial state, Eq. (23). We have already seen that this form is not unique in the sense that different choices of $\hat{A}$ can give rise to either LT or non-LT VER pathways. At the same time, it is conceivable that other types of initial states can give rise to VER of the LT-type. Furthermore, it may be possible to find an initial state that will lead to an expression for the VER rate constant that reduces to the LT formula under the conditions of validity of BRT and can be obtained directly from CMD simulations. That this fascinating possibility can be realized is shown in the present section.

We start out by considering the following, completely general, form of the initial state of the overall system:

$$
\hat{\rho}(0)=\frac{e^{-\beta \hat{H}}}{Z}+\hat{\Delta}
$$

The deviation from equilibrium, $\hat{\Delta}$, must obviously satisfy $\operatorname{Tr}(\hat{\Delta})=0, \hat{\Delta}^{\dagger}=\hat{\Delta}$, and keep $\hat{\rho}(0)$ positive. Following a similar procedure to that discussed in the previous section, leads to the following general expression for the relaxation of $\left\langle\hat{H}_{s}\right\rangle$ to equilibrium:

$$
k=\frac{\alpha}{\mu} \frac{\int_{0}^{\beta} d \beta_{1} \int_{0}^{\beta_{1}} d \beta_{2}\left\langle\delta \hat{q}\left(-i \beta_{1} / \hbar\right) \delta \hat{q}\left(-i \beta_{2} / \hbar\right)[\hat{p} \hat{F}](t)\right\rangle_{\mathrm{eq}}}{\int_{0}^{\beta} d \beta_{1} \int_{0}^{\beta_{1}} d \beta_{2}\left\langle\delta \hat{q}\left(-i \beta_{1} / \hbar\right) \delta \hat{q}\left(-i \beta_{2} / \hbar\right) \delta \hat{H}_{s}\right\rangle_{\mathrm{eq}}} .
$$

It should be noted that Eq. (31) applies to any initial state, regardless of its proximity to equilibrium, and is therefore more general than standard LRT. The latter can obviously be retrieved from Eq. (31) by substituting $\hat{\Delta}=-f \int_{0}^{\beta} d \lambda e^{-(\beta-\lambda) \hat{H}} \delta \hat{A} e^{-\lambda \hat{H}} / Z$. Finally, assuming that $\left\langle\delta \hat{H}_{s}\right\rangle(t)$ follows rate kinetics, one can put the VER rate constant in the following form:

$$
k=\frac{\alpha}{\mu} \frac{\operatorname{Tr}\{\hat{\Delta}[\hat{p} \hat{F}](t)\}}{\operatorname{Tr}\left\{\hat{\Delta} \delta \hat{H}_{s}\right\}} .
$$

It should be noted that the actual validity of rate kinetics, as well as the value of the rate constant, may depend on the choice of $\hat{\Delta}$.

We now consider the following particular choice of $\hat{\Delta}$ :

$$
\begin{aligned}
\hat{\Delta}= & f^{2} \frac{e^{-\beta \hat{H}}}{Z} \int_{0}^{\beta} d \beta_{1} \int_{0}^{\beta_{1}} d \beta_{2}\left[\delta \hat{q}\left(-i \beta_{1} / \hbar\right)\right. \\
& \left.\times \delta \hat{q}\left(-i \beta_{2} / \hbar\right)-\left\langle\delta \hat{q}\left(-i \beta_{1} / \hbar\right) \delta \hat{q}\left(-i \beta_{2} / \hbar\right)\right\rangle_{\mathrm{eq}}\right] .
\end{aligned}
$$

This $\hat{\Delta}$ obviously corresponds to the second-order term in the expansion of the original initial state, Eq. (23), in powers of $f$. As such, it must satisfy $\operatorname{Tr}(\hat{\Delta})=0, \hat{\Delta}^{\dagger}=\hat{\Delta}$. However, it is important to note that using an initial state in the form of Eq. (30), with $\hat{\Delta}$ as in Eq. (33), is not the same as expanding $\hat{\rho}(0)$ in Eq. (23) to second order in $f$, since the first-order term is now missing. Keeping this first-order term out is important for obtaining an expression for the VER rate constant which is independent of $f$ [including Eq. (33) as a second order term in the expansion of Eq. (23) would correspond to nonlinear, rather than linear, response theory].

Upon substitution of Eq. (33) into Eq. (32), we obtain the following expression for the VER rate constant:

It is important to note that the correlation function in the numerator of Eq. (34) can be obtained directly from CMD simulations [cf. Eq. (12)]. Furthermore, the fact that $\hat{\Delta}$ is quadratic in $\delta \hat{q}$ suggests that this initial state may relax via a LT-type pathway. This indeed turns out to be the case. More specifically, one can show that $k$ in Eq. (34) reduces to $1 / T_{1}$ of the LT formula, Eq. (18), upon imposing the three basic assumptions underlying BRT. The actual proof, which is rather tedious, is outlined in Appendix A.

\section{Derivation of the centroid Landau-Teller formula}

As mentioned above, the correlation function in the numerator of Eq. (34) is directly related to the corresponding centroid correlation function [cf. Eq. (12)]. Thus, by only imposing the CMD approximation, Eq. (11), on the centroid symbol of the time-dependent energy flux operator in Eq. (34), we can obtain the following working expression for the VER rate constant: 


$$
\begin{aligned}
k_{\mathrm{CMD}}= & \frac{\alpha}{\mu} \int d q_{c} \int d p_{c} \int d \mathbf{Q}_{\mathbf{c}} \int d \mathbf{P}_{\mathbf{c}} \exp \left\{-\beta\left[\sum_{i=1}^{N} \frac{\left(P_{c}^{(i)}\right)^{2}}{2 M^{(i)}}+\frac{p_{c}^{2}}{2 m}+U_{c m}\left(\mathbf{Q}_{\mathbf{c}}, q_{c}\right)\right]\right\}\left[\delta q_{c}(-t)\right]^{2} p_{c} F_{c}\left(\mathbf{Q}_{\mathbf{c}}, q_{c}\right) \\
& \times\left\{\int d q_{c} \int d p_{c} \int d \mathbf{Q}_{\mathbf{c}} \int d \mathbf{P}_{\mathbf{c}} \exp \left\{-\beta\left[\sum_{i=1}^{N} \frac{\left(P_{c}^{(i)}\right)^{2}}{2 M^{(i)}}+\frac{p_{c}^{2}}{2 m}+U_{c m}\left(\mathbf{Q}_{\mathbf{c}}, q_{c}\right)\right]\right\}\left[\delta q_{c}\right]^{2}\left[\delta H_{s}\right]_{c}\left(q_{c}, p_{c}, \mathbf{Q}_{\mathbf{c}}, \mathbf{P}_{\mathbf{c}}\right)\right\}^{-1},
\end{aligned}
$$

where

$$
U_{c m}\left(\mathbf{Q}_{\mathbf{c}}, q_{c}\right)=\mu \omega^{2} q_{c}^{2} / 2+V_{c m}\left(\mathbf{Q}_{\mathbf{c}}, q_{c}\right)+\alpha q_{c} F_{c}\left(\mathbf{Q}_{\mathbf{c}}, q_{c}\right) .
$$

In arriving at Eq. (35), we have made use of the classical-like time-reversal symmetry of the CMD correlation function $\left(\left\langle A_{c} B_{c}(t)\right\rangle_{\mathrm{CMD}}=\left\langle A_{c}(-t) B_{c}\right\rangle_{\mathrm{CMD}}\right)$. We have also used the centroid symbol of the energy flux operator, Eq. (28), which can be derived from Eq. (3) (see also Ref. 89 for the path integral expression for $\hat{\phi}$, and Ref. 105 for an explicit multi-dimensional presentation of the centroid formalism). The derivation is straightforward, although rather tedious, and leads to the following final result:

$$
\left[\hat{H}_{s}\right]_{c}=-\frac{\alpha}{\mu}[\hat{p} F(\hat{\mathbf{Q}})]_{c}=-\frac{\alpha}{\mu} p_{c} F_{c}\left(\mathbf{Q}_{\mathbf{c}}, q_{c}\right),
$$

where, $\mathbf{Q}_{\mathbf{c}}=\left\{Q_{c}^{(1)}, \ldots, Q_{c}^{(N)}\right\}$, and

$$
\begin{aligned}
F_{c}\left(\mathbf{Q}_{\mathbf{c}}, q_{c}\right)= & \left\{\int_{q(0)=q(\beta \hbar)} D q(\lambda) \int_{\mathbf{Q}(0)=\mathbf{Q}(\beta \hbar)} D \mathbf{Q}(\lambda) \delta\left[q_{c}-\int_{0}^{\beta \hbar} \frac{d \lambda}{\beta \hbar} q(\lambda)\right] \delta\left[\mathbf{Q}_{\mathbf{c}}-\int_{0}^{\beta \hbar} \frac{d \lambda}{\beta \hbar} \mathbf{Q}(\lambda)\right]\right. \\
& \left.\times \int_{0}^{\beta \hbar} \frac{d \lambda}{\beta \hbar} F[\mathbf{Q}(\lambda)] \exp \{-\mathcal{S}[q(\lambda), \mathbf{Q}(\lambda)] / \hbar\}\right\}\left\{\int_{q(0)=q(\beta \hbar)} D q(\lambda) \int_{\mathbf{Q}(0)=\mathbf{Q}(\beta \hbar)} D \mathbf{Q}(\lambda)\right. \\
& \left.\times \delta\left[q_{c}-\int_{0}^{\beta \hbar} \frac{d \lambda}{\beta \hbar} q(\lambda)\right] \delta\left[\mathbf{Q}_{\mathbf{c}}-\int_{0}^{\beta \hbar} \frac{d \lambda}{\beta \hbar} \mathbf{Q}(\lambda)\right] \exp \{-\mathcal{S}[q(\lambda), \mathbf{Q}(\lambda)] / \hbar\}\right\}^{-1} \\
= & \lim _{P \rightarrow \infty}\left\{\int d q_{1} \cdots \int d q_{P} \int d \mathbf{Q}_{1} \cdots \int d \mathbf{Q}_{\mathbf{P}} \delta\left[q_{c}-\sum_{k=1}^{P} \frac{q_{k}}{P}\right] \delta\left[\mathbf{Q}_{\mathbf{c}}-\sum_{k=1}^{P} \frac{\mathbf{Q}_{\mathbf{k}}}{P}\right]\right. \\
& \left.\times\left[\sum_{k=1}^{P} \frac{F\left(\mathbf{Q}_{\mathbf{k}}\right)}{P}\right] \exp \left\{-\mathcal{S}\left(q_{1}, \ldots, q_{P}, \mathbf{Q}_{\mathbf{1}}, \ldots, \mathbf{Q}_{\mathbf{P}}\right) / \hbar\right\}\right\}\left\{\int d q_{1} \cdots \int d q_{P} \int d \mathbf{Q}_{\mathbf{1}} \cdots \int d \mathbf{Q}_{\mathbf{P}} \delta\left[q_{c}-\sum_{k=1}^{P} \frac{q_{k}}{P}\right]\right. \\
& \left.\times \delta\left[\mathbf{Q}_{\mathbf{c}}-\sum_{k=1}^{P} \frac{\mathbf{Q}_{\mathbf{k}}}{P}\right] \exp \left\{-\mathcal{S}\left(q_{1}, \ldots, q_{P}, \mathbf{Q}_{\mathbf{1}}, \ldots, \mathbf{Q}_{\mathbf{P}}\right) / \hbar\right\}\right\},
\end{aligned}
$$

with

$$
\begin{aligned}
\frac{1}{\hbar} \mathcal{S}[q(\lambda), \mathbf{Q}(\lambda)] \\
=\lim _{P \rightarrow \infty} \frac{1}{\hbar} \mathcal{S}\left[q_{1}, \ldots, q_{P}, \mathbf{Q}_{\mathbf{1}}, \ldots, \mathbf{Q}_{\mathbf{P}}\right] \\
=\frac{1}{\hbar} \int_{0}^{\beta \hbar} d \lambda\left\{\frac{1}{2} m[\dot{q}(\lambda)]^{2}+\sum_{i=1}^{N} \frac{1}{2} M^{(i)}\left[\dot{Q}^{(i)}(\lambda)\right]^{2}\right. \\
\left.\quad+\frac{1}{2} \mu \omega q^{2}(\lambda)\right)+V[\mathbf{Q}(\lambda)]+\alpha q(\lambda) F[\mathbf{Q}(\lambda)],
\end{aligned}
$$

$$
\begin{aligned}
\frac{1}{\hbar} \mathcal{S}[ & \left.q_{1}, \ldots, q_{P}, \mathbf{Q}_{1}, \ldots, \mathbf{Q}_{\mathbf{P}}\right] \\
= & \beta \sum_{k=1}^{P}\left\{\frac{1}{2} \mu \omega_{P}^{2}\left(q_{k}-q_{k+1}\right)^{2}\right. \\
& +\sum_{i=1}^{N} \frac{1}{2} M^{(i)} \omega_{P}^{2}\left(Q_{k}^{(i)}-Q_{k+1}^{(i)}\right)^{2} \\
& \left.+\frac{1}{P}\left(\frac{1}{2} \mu \omega q_{k}^{2}+V\left(\mathbf{Q}_{\mathbf{k}}\right)+\alpha q_{k} F\left[\mathbf{Q}_{\mathbf{k}}\right]\right)\right\},
\end{aligned}
$$$$
q_{1}=q_{P+1}, \mathbf{Q}_{\mathbf{P}+\mathbf{1}}=\mathbf{Q}_{\mathbf{1}} \text {, and } \omega_{p}^{2}=P /(\beta \hbar)^{2} \text {. }
$$

$F_{c}\left(q_{c}, \mathbf{Q}_{\mathbf{c}}\right)$ can be obtained from PIMD or PIMC simu- 
lations of a system consisting of $N+1$ chain polymers, whose centroids are fixed at $\left(q_{c}, Q_{c}^{(1)}, \ldots, Q_{c}^{(N)}\right)$. More specifically, $F_{c}\left(q_{c}, \mathbf{Q}_{\mathbf{c}}\right)$ corresponds to the average force exerted on the beads of the polymer chain associated with vibrational mode, $\left\{q_{1}, \ldots, q_{P}\right\}$, by the polymer chains associated with the bath DOF, $\left\{\mathbf{Q}_{1}, \ldots, \mathbf{Q}_{\mathbf{P}}\right\}$. It is important to note that the centroid symbol of the force is distinctly different from the classical force for the corresponding centroid position, i.e., $F_{c}\left(q_{c}, \mathbf{Q}_{\mathbf{c}}\right) \neq F\left(\mathbf{Q}_{\mathbf{c}}\right)$.

In principle, one could evaluate the VER rate constant (if it exists) directly from Eq. (35). However, in this paper, we are primarily interested in VER under the conditions of validity of the LT formula. Thus, we proceed by imposing the three basic assumptions underlying BRT upon the VER rate constant in Eq. (35). The details of the analysis are left to Appendix B, and only the final result is given below:

$$
k_{\mathrm{CMD}} \stackrel{\mathrm{BRT}}{\rightarrow} \frac{1}{T_{1}^{\mathrm{CMD}}}=\alpha^{2} \frac{\beta}{2 \mu} \widetilde{C}^{\mathrm{CMD}}(\omega) \text {, }
$$

where $\widetilde{C}^{\mathrm{CMD}}(\omega)$ is the FT of

$$
C^{\mathrm{CMD}}(\tau)=\frac{\int d \mathbf{Q}_{\mathbf{c}} \int d \mathbf{P}_{\mathbf{c}} \exp \left\{-\beta\left[\sum_{i=1}^{N} \frac{\left(P_{c}^{(i)}\right)^{2}}{2 M^{(i)}}+V_{c m}\left(\mathbf{Q}_{\mathbf{c}}\right)\right]\right\} \delta F_{c}\left[\mathbf{Q}_{\mathbf{c}}(\tau)\right] \delta F_{c}\left[\mathbf{Q}_{\mathbf{c}}\right]}{\int d \mathbf{Q}_{\mathbf{c}} \int d \mathbf{P}_{\mathbf{c}} \exp \left\{-\beta\left[\sum_{i=1}^{N} \frac{\left(P_{c}^{(i)}\right)^{2}}{2 M^{(i)}}+V_{c m}\left(\mathbf{Q}_{\mathbf{c}}\right)\right]\right\}},
$$

$F_{c}\left[\mathbf{Q}_{\mathbf{c}}\right] \equiv F_{c}\left[\mathbf{Q}_{\mathbf{c}}, q_{c}=0\right]$, and $\mathbf{Q}_{\mathbf{c}}(\tau)$ follows a classical time evolution which is governed by the centroid potential $V_{c m}\left(\mathbf{Q}_{\mathbf{c}}\right) \equiv V_{c m}\left(\mathbf{Q}_{\mathbf{c}}, q_{c}=0\right)$.

It should be noted that $C^{\mathrm{CMD}}(\tau)$ has the same form as the classical FFCF. Quantum effects are introduced in two distinct ways: (1) The initial sampling and the dynamics are governed by the free bath centroid potential, $V_{c m}\left(\mathbf{Q}_{\mathbf{c}}\right)$, rather than the classical potential, $V\left(\mathbf{Q}_{\mathbf{c}}\right)$; (2) The classical force, $F\left(\mathbf{Q}_{\mathbf{c}}\right)$, is replaced by the corresponding centroid symbol of the force, $F_{c}\left(\mathbf{Q}_{\mathbf{c}}\right)$. Thus, the classical limit of $C^{\mathrm{CMD}}(\tau)$ obviously coincides with the exact classical limit, which is not always the case in some of the other centroid-based approaches, that also involve additional approximations. At the same time, $C^{\mathrm{CMD}}(\tau)$ does not coincide with the exact quantum result at $\tau=0$. In this context, it is important to emphasize that the time integration variable $\tau$ in Eq. (42) has nothing to do with the explicit time variable $t$ in Eq. (34). More specifically, one should distinguish between $C^{\mathrm{CMD}}(\tau)$ and the centroid correlation function in our original expression, Eq. (34). The latter is directly related to the corresponding Kubo-transformed correlation function and is therefore exact at $t=0$. However, the actual VER rate constant is related to the behavior of this correlation function at long times ( $t$ $\gg k^{-1}$ ). In fact, Eq. (41) is independent of $t$, and one cannot take its $t=0$ limit since the long-time limit is already incorporated into it as part of the conditions underlying BRT (as shown in Appendix B, the $\pm \infty$ in the limits of the Fourier integral originate from the assumption that $t \sim T_{1} \gg \tau_{c}$ ).

Finally, we note that it would be more appropriate to interpret Eq. (41) as an approximation for the VER rate constant, rather than interpret $C^{\mathrm{CMD}}(\tau)$, Eq. (42), as an approximation for the quantum-mechanical FFCF. The reason for this is that Eq. (41) originates from Eq. (35), which provides an approximation for the VER rate constant in terms of another correlation function. In fact, treating Eq. (41) as an approximation for the exact LT formula, Eq. (18), gives rise the following approximation for the exact quantummechanical FFCF and its FT:

$$
\begin{aligned}
& C(\tau) \approx \int_{-\infty}^{\infty} d \omega e^{-i \omega \tau} \frac{\beta \hbar \omega}{1-e^{-\beta \hbar \omega}} \widetilde{C}^{\mathrm{CMD}}(\omega), \\
& \widetilde{C}(\omega) \approx \frac{\beta \hbar \omega}{1-e^{-\beta \hbar \omega}} \widetilde{C}^{\mathrm{CMD}}(\omega) .
\end{aligned}
$$

Inspection of Eq. (44) reveals that it coincides with the approximation based on the harmonic QCF (cf. Table II) if $\widetilde{C}^{\mathrm{CMD}}(\omega)$ is replaced with the FT of the classical FFCF, $\widetilde{C}^{\mathrm{Cl}}(\omega)$. Thus, the approximate VER rate constant of Eq. (41) naturally incorporates the harmonic QCF and adds to it quantum corrections that originate from the difference between $C^{\mathrm{CMD}}(\tau)$ and $C^{\mathrm{Cl}}(\tau)$. It should also be noted that $C(\tau)$ in Eq. (43) is complex, with real and imaginary parts.

\section{APPLICATIONS}

In this section, we consider the application of Eq. (41) for calculating the VER rate constant in three model systems: (1) A harmonic vibrational mode coupled to a harmonic bath with a force which is an exponential function of the bath coordinates; (2) A diatomic molecule coupled to a short chain of Helium atoms with nearest neighbor Lennard-Jones (LJ) interactions; (3) A breathing sphere diatomic molecule in a monoatomic solvent with LJ interactions. The parameters are chosen to be the same as in Ref. 133, and the corresponding LHA-LSC-IVR-based results are presented alongside the CMD-based predictions, for the sake of comparison. The purpose of this analysis is to critically examine the ability of the CMD approximation, upon which Eq. (41) is based, to quantitatively capture the large quantum enhancement of VER rate constants. It should be noted that previous studies seem to suggest that CMD, supplemented by additional approximations, is indeed useful for describing quantum-mechanical VER. In our case, we avoided such additional approximations, which enables us to perform a clean test of just the CMD approximation. 


\section{A. Exponential coupling to a harmonic bath}

The first model that we consider involves a bath consisting of uncoupled harmonic oscillators of different frequencies,

$$
\hat{H}_{b}=\sum_{j=1}^{N}\left(\frac{\left(\hat{P}^{(j)}\right)^{2}}{2 M^{(j)}}+\frac{1}{2} M^{(j)}\left(\omega^{(j)}\right)^{2}\left(\hat{Q}^{(j)}\right)^{2}\right),
$$

and a force which is exponential in the bath coordinates,

$$
F(\hat{\mathbf{Q}})=e^{R(\hat{\mathbf{Q}})},
$$

where

$$
R(\hat{\mathbf{Q}})=\sum_{j} c^{(j)} \sqrt{\frac{2 M^{(j)} \omega^{(j)}}{\hbar}} \hat{Q}^{(j)} .
$$

The fact that the exact quantum-mechanical FFCF can be obtained analytically for this model ${ }^{42}$ has established it as a convenient benchmark. $^{51,65}$ The exact quantum-mechanical FFCF is given by

$$
C(\tau)=e^{B(0)}\left(e^{B(\tau)}-1\right),
$$

where

$$
\begin{aligned}
& B(\tau)=\left\langle\hat{R}_{0}(\tau) \hat{R}(0)\right\rangle_{0}= \int_{0}^{\infty} d \omega \Gamma(\omega)\left\{[n(\omega)+1] e^{-i \omega \tau}\right. \\
&\left.+n(\omega) e^{i \omega \tau}\right\} \\
& \Gamma(\omega)=\sum_{k}\left(c^{(k)}\right)^{2} \delta\left(\omega-\omega^{(k)}\right)
\end{aligned}
$$

and $n(\omega)=[\exp (\beta \hbar \omega)-1]^{-1}$. The calculations reported below were performed with a spectral density of the following form:

$$
\Gamma(\omega)=2 \lambda \frac{\omega^{\alpha}}{\omega_{c}^{\alpha+1}} \exp \left(-\frac{\omega^{2}}{\omega_{c}^{2}}\right),
$$

and for the following values of the parameters: $\lambda=0.20, \alpha$ $=3$, and $\beta \hbar \omega_{c}=4.0$.

The fact that the bath is harmonic in this case, made it possible to establish a simple analytical relationship between the classical force and its centroid symbol. The details of the derivation are left to Appendix $\mathrm{C}$, and only the final result is given below,

$$
F_{c}\left(\mathbf{Q}_{\mathbf{c}}\right)=\zeta F\left(\mathbf{Q}_{\mathbf{c}}\right),
$$

where

$$
\begin{aligned}
\zeta & =\frac{\langle F(\hat{\mathbf{Q}})\rangle_{0}}{\langle F(\mathbf{Q})\rangle_{0}^{\mathrm{Cl}}} \\
& =\exp \left\{\sum_{k=1}^{N}\left(c^{(k)}\right)^{2}\left[\operatorname{coth}\left(\beta \hbar \omega^{(k)} / 2\right)-\left(\beta \hbar \omega^{(k)} / 2\right)^{-1}\right]\right\}>1 .
\end{aligned}
$$

Since the bath is harmonic, the centroid potential, $V_{c m}\left(\mathbf{Q}_{\mathbf{c}}\right)$, coincides in this case with the classical potential, $V\left(\mathbf{Q}_{\mathbf{c}}\right)$. This implies that $C^{\mathrm{CMD}}(\tau)=\zeta^{2} C^{\mathrm{Cl}}(\tau)$ and

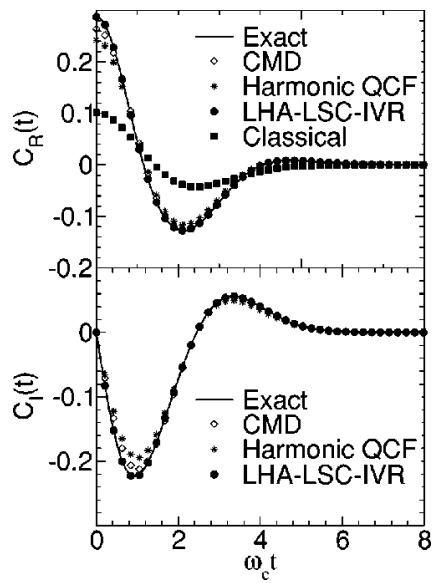

FIG. 1. The real and imaginary parts of the FFCF, for the case of exponential coupling to a harmonic bath. The exact, CMD-based, harmonic-QCFbased, LHA-LSC-IVR-based and classical results are shown.

$$
\frac{1}{T_{1}^{\mathrm{CMD}}}=\zeta^{2} \frac{1}{T_{1}^{\mathrm{Cl}}} .
$$

Thus, one may think of $\zeta^{2}$ as an additional QCF that enhances the quantum rate constant over the classical one, and supplements the already built-in harmonic QCF. However, the fact that $\zeta^{2}$ is independent of the frequency $\omega$, immediately implies that this factor will not be able to account for the rapid growth in the quantum correction factor, expected with increasing frequency.

The real and imaginary parts of the FFCF, obtained from Eq. (43), are shown in Fig. 1. Also shown in Fig. 1 are the exact, classical and LHA-LSC-IVR-based results, as well as a prediction based on the harmonic QCF. The CMD-based FFCF is somewhat better than the one based on the harmonic QCF, which should be attributed to the $\zeta^{2}$ factor. Furthermore, the CMD-based FFCF is seen to be in reasonable agreement with the exact FFCF, although it should be noted that much of the improvement over the classical result is due to the inherent harmonic QCF, and not the $\zeta^{2}$ factor.

Despite the reasonable agreement in the time-domain, one must not lose sight of the fact that VER is dominated by the high-frequency tail of the FFCF in the frequency-domain. A plot of the the FT of the FFCF, as predicted by Eq. (44), is given in Fig. 2, in regular and semilog formats. The regular plot suggests that the CMD-based result is in good agreement with the exact result. It is also significantly better than the harmonic-QCF-based result, which is once again attributed to the $\zeta^{2}$ factor. Thus, CMD does seem to capture the bulk of the power spectrum rather well, although much of this effect should actually be attributed to the inherent harmonic QCF and is not directly related to CMD. However, the semilog plot shows that the high-frequency tail of this power spectrum, which is relevant for VER, essentially coincide with the harmonic-QCF-based result. Thus, the modifications due to the $\zeta^{2}$ factor, which are directly associated with CMD, are rather small at the high-frequency domain. It should also be noted that the LHA-LSC-IVR-based result is in excellent agreement with the exact result in this case, and is signifi- 


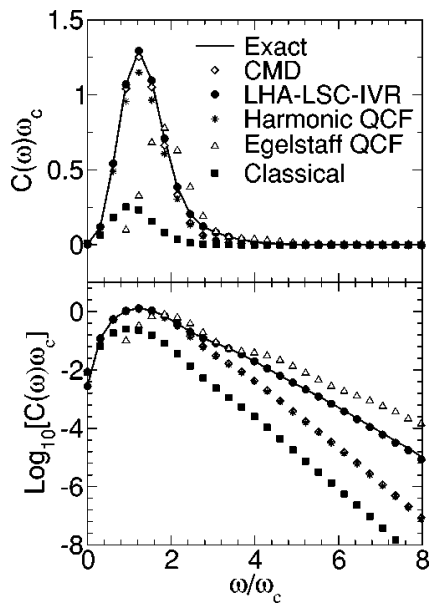

FIG. 2. The power spectrum of the FFCF, for the case of exponential coupling to a harmonic bath, in regular (upper panel) and semilog (lower panel) formats. The exact, CMD-based, LHA-LSC-IVR-based, harmonic-QCFbased, Egelstaff-QCF-based, and classical results are shown.

cantly better than the harmonic-QCF-based and CMD-based predictions.

The inability of CMD to provide a significant improvement over the harmonic QCF is rather surprising in light of the fact that a seemingly more approximate method, which supplements the CMD approximation with an approximate second-order cumulant expansion (C-CMD) of the FFCF, has been known to yield the exact result for this model ${ }^{84,103}$ In this case, one expresses the FFCF in terms of the position autocorrelation function, which can be obtained exactly from CMD when the system is harmonic. However, this fortunate coincidence is clearly restricted to harmonic systems, and it is not clear how well it will hold in highly anharmonic systems such as liquids. Furthermore, the average position, which is essential for calculating the position autocorrelation function, is ill-defined in simulations of liquids with periodic boundary conditions (previous applications of C-CMD were restricted to cases where the relaxing molecule was held fixed). Thus, the fact that C-CMD, which is more approximate than the method presented herein, happens to be exact for this specific model, should probably not be interpreted as implying that CMD is generally applicable to high-frequency VER.

\section{B. A linear helium cluster}

The second model to be considered has been recently used by Poulsen and co-workers ${ }^{46,103,104}$ for calculating the FFCF via the C-CMD method. In this model, a harmonic diatomic molecule, $A_{2}$, is attached to a wall, and held fixed perpendicular to it (cf. Fig. 3). The $A$ atom which is not attached to the wall is coupled to a short linear chain of four helium atoms, with the last helium atom held in place. The interaction between the $A$ atom and the helium atom next to it, as well as the interactions between the helium atoms, are described by anharmonic Lennard-Jones (LJ) potentials, which mimic realistic liquid-phase interactions. Only nearest neighbor interactions are taken into account.

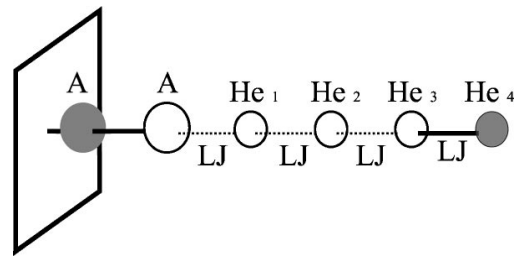

FIG. 3. A schematic view of the linear helium cluster model.

The $A_{2}$ molecule and helium chain are assumed to lie along the $\mathrm{x}$ axis, with the origin positioned on the $A$ atom which is attached to the wall. The second $A$ atom is positioned at $x_{0}=r_{\mathrm{eq}}+\delta_{0}$, where $r_{\mathrm{eq}}$ is the equilibrium bond length of $A_{2}$ and $\delta_{0}$ is the deviation relative to it. The positions of Helium atoms 1,2 , and 3 are given by $x_{1}=r_{\mathrm{eq}}$ $+\sigma_{\mathrm{He}-A}+\delta_{1}, \quad x_{2}=r_{\mathrm{eq}}+\sigma_{\mathrm{He}-A}+\sigma_{\mathrm{He}-\mathrm{He}}+\delta_{2}$ and $x_{3}=r_{\mathrm{eq}}$ $+\sigma_{\mathrm{He}-A}+2 \sigma_{\mathrm{He}-\mathrm{He}}+\delta_{3}$, respectively, where $\sigma_{\mathrm{He}-A}$ and $\sigma_{\mathrm{He}-\mathrm{He}}$ are the familiar LJ parameters. The position of the last Helium atom is fixed at $x_{4}=r_{\mathrm{eq}}+\sigma_{\mathrm{He}-\mathrm{A}}+3 \sigma_{\mathrm{He}-\mathrm{He}}$. The overall potential energy of this system is given by

$$
\begin{aligned}
V\left(\delta_{0}, \delta_{1}, \delta_{2}, \delta_{3}\right)= & \frac{1}{2} \mu \omega^{2} \delta_{0}^{2}+V_{\mathrm{LJ}}^{\mathrm{He}-A}\left(\sigma_{\mathrm{He}-A}+\delta_{1}-\delta_{0}\right) \\
& +V_{\mathrm{LJ}}^{\mathrm{He}-\mathrm{He}}\left(\sigma_{\mathrm{He}-\mathrm{He}}+\delta_{2}-\delta_{1}\right) \\
& +V_{\mathrm{LJ}}^{\mathrm{He}-\mathrm{He}}\left(\sigma_{\mathrm{He}-\mathrm{He}}+\delta_{3}-\delta_{2}\right) \\
& +V_{\mathrm{LJ}}^{\mathrm{He}-\mathrm{He}}\left(\sigma_{\mathrm{He}-\mathrm{He}}-\delta_{3}\right)
\end{aligned}
$$

where $V_{\mathrm{LJ}}(r)=4 \epsilon\left[(\sigma / r)^{12}-(\sigma / r)^{6}\right]$. The parameters used are the same as these in Ref. 103: $\sigma_{\mathrm{He}-A}=4.944$ a.u., $\sigma_{\mathrm{He}-\mathrm{He}}=4.310$ a.u., $\quad \epsilon_{\mathrm{He}-A} / k_{B}=25.1 \mathrm{~K}, \quad \epsilon_{\mathrm{He}-\mathrm{He}} / k_{B}$ $=10.2 \mathrm{~K}$. The force on the diatomic molecule is obtained by linearizing the potential with respect to the diatomic displacement, $\delta_{0}$;

$$
F=-\left.\frac{\partial V}{\partial \delta_{0}}\right|_{\delta_{0}=0}=V_{\mathrm{LJ}}^{\prime \mathrm{He}-A}\left(\sigma_{\mathrm{He}, A}+\delta_{1}\right) .
$$

The real part of the FFCF, obtained from Eq. (43), is shown in Fig. 4 (note that $\langle F(\tau) F\rangle$ is plotted, rather than $\langle\delta F(\tau) \delta F\rangle$ ). Also shown are the exact, classical, harmonicQCF-based, LHA-LSC-IVR-based and C-CMD-based re-

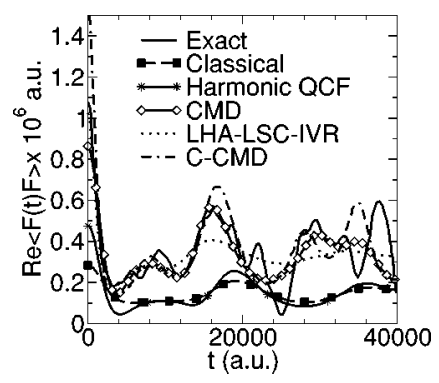

FIG. 4. The real part of the (nonshifted) FFCF, as obtained for the Helium cluster model. Shown are the exact, classical, harmonic-QCF-based, CMDbased, LHA-LSC-IVR-based, and C-CMD-based results. 
sults (the exact and C-CMD-based results were adopted from Ref. 103). The relatively large deviation between the classical and exact quantum-mechanical results is indicative of the fact that the "solvent" is pronouncedly quantum-mechanical in this case, as could be expected from helium at $40 \mathrm{~K}$. The failure of the harmonic QCF also suggests that this system is pronouncedly anharmonic. The prediction based on Eq. (43) is seen to provide a rather reasonable approximation of the exact quantum-mechanical FFCF in this case. This reinforces the notion that CMD is capable of providing a reasonable approximation for the time-domain behavior of a pronouncedly quantum-mechanical and anharmonic system. The agreement between the C-CMD-based prediction and the exact result is clearly not as good, in accord with the more approximate nature of C-CMD. Finally, it is interesting to compare the prediction obtained from CMD to that obtained via LHA-LSC-IVR. Generally speaking, Eq. (44) seem to provide a better approximation to the exact FFCF over the time interval considered. This nicely reflects the fact that the dynamics in LHA-LSC-IVR is purely classical, whereas the dynamics in CMD includes quantum effects via the centroid potential. However, LHA-LSC-IVR is exact at $t=0$ and clearly provides a more accurate description of the initial decay of the FFCF. Noting that correlation functions are often found to be short-lived in truly condensed phase systems, and that their high-frequency FT is dominated by this short time decay, then leads one to expect that LHA-LSC-IVR would be better suited than CMD for calculating highfrequency VER rate constants.

\section{A diatomic solute in a monoatomic solvent (breathing sphere model)}

We next consider the VER of a diatomic solute in a monoatomic solvent. The vibrational mode is assumed to have a spherical symmetry, and can therefore be viewed as a "breathing sphere." 43,150,151 The solute-solvent and solvent-solvent interactions are treated in terms of spherically symmetric pair potentials. The overall potential energy is given by

$$
V=\frac{1}{2} \mu \omega^{2} q^{2}+\sum_{j<k} \phi_{s}\left(r_{j k}\right)+\sum_{j} \phi\left(r_{j 0}, q\right),
$$

where $\phi_{s}(r)$ is the solvent-solvent pair potential, $\phi(r, q)$ is the solvent-solute pair potential, $r_{j k}$ is the distance between the $j$ th and $k$ th solvent atoms, and $r_{j 0}$ is the distance between the center of mass of the diatomic solute and the $j$ th solvent atom. The force is obtained by the linearization of the potential with respect to $q$,

$$
F=-\left.\frac{\partial V}{\partial q}\right|_{q=0}=-\left.\sum_{j} \frac{\partial \phi}{\partial q}\right|_{q=0}\left(r_{j 0}\right) .
$$

In order to enhance the computational feasibility, calculations were performed on a two-dimensional liquid and under the assumption that the solvent atoms and the diatomic solute

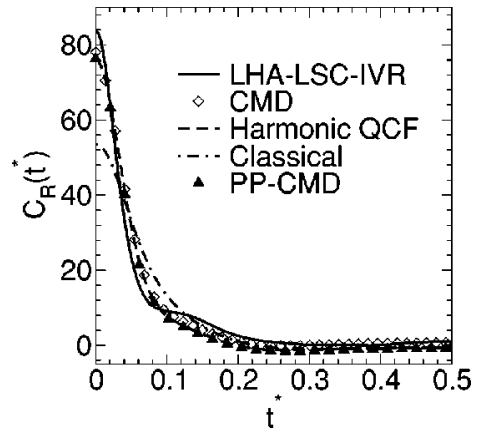

FIG. 5. The real part of the FFCF, as obtained for the breathing sphere neon model. Shown are the LHA-LSC-IVR-based, CMD-based, PP-CMD-based, harmonic-QCF-based, and classical results.

have the same mass, and that $\phi_{s}(r)$ and $\phi(r, q=0)$ are identical and given by a LJ potential, ${ }^{152} \phi_{s}(r)=\phi(r, q=0)$ $=V_{\mathrm{LJ}}(r)=4 \epsilon\left[(\sigma / r)^{12}-(\sigma / r)^{6}\right]$.

In Figs. 5 and 6, we show the FFCF and its FT, as obtained from simulations where the mass and $\mathrm{LJ}$ parameters were chosen to coincide with these of liquid neon, namely, $\sigma=2.72 \AA$ and $\epsilon / k_{B}=47.0 \mathrm{~K}$. The calculations were performed on a 2D liquid consisting of 81 atoms confined to a square, at a reduced density and temperature of $\rho^{*}=0.70$ $\left(\rho=9.46 \mathrm{~nm}^{-2}\right)$ and $T^{*}=0.68(T=32 \mathrm{~K})$, respectively. Periodic boundary conditions and a potential cutoff at $3 \sigma$ have been employed. The real part of the FFCF, as obtained from Eq. (43), is shown in Fig. 5. Since the exact quantummechanical result is not known for this case, and experimental results are unavailable for this particular model system, evaluation of the quality of the approximation is difficult. However, Eq. (43) yields a FFCF which is clearly different from the classical one, and close to the one obtained via LHA-LSC-IVR. However, it is important to note that this difference arises almost exclusively from the harmonic QCF. The FT of the FFCF, as obtained from Eq. (44), is shown in Fig. 6 on a semilog plot. The high-frequency tail of Eq. (44) clearly coincides with that obtained via the harmonic QCF. Thus, in this case, the fact that we used CMD instead of classical MD did not make a difference (as long as we applied the harmonic QCF to the latter). It should also be noted that the prediction of LHA-LSC-IVR, which has been seen to be very reliable in other models, ${ }^{133}$ deviates considerably from that based on CMD or the harmonic QCF.

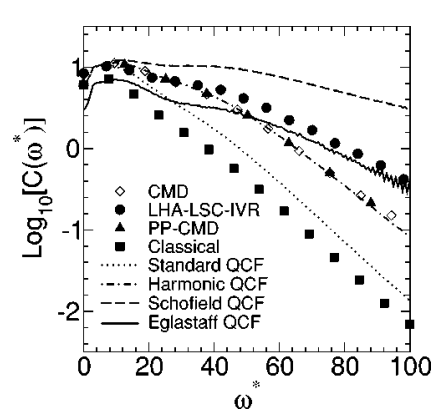

FIG. 6. A semilog plot of the FT of the FFCF for the breathing sphere neon model. Shown are results obtained from CMD, PP-CMD, LHA-LSC-IVR, classical mechanics, and the standard, harmonic, Schofield and Egelstaff QCFs. 


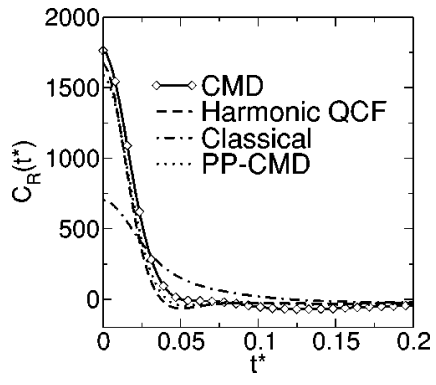

FIG. 7. The real part of the FFCF, as obtained for the breathing sphere Helium model. Shown are the CMD-based, PP-CMD-based, harmonicQCF-based, and classical results.

In Figs. 7 and 8, we show the FFCF and its FT, as obtained from simulations, where the mass and LJ parameters were chosen to coincide with these of liquid helium (the LJ parameters were adopted from Sec. IV B). The calculations were performed on a 2D liquid consisting of 81 atoms confined to a square, at a reduced density and temperature of $\rho^{*}=0.70\left(\rho=13.46 \mathrm{~nm}^{-2}\right)$ and $T^{*}=3.92(T=40 \mathrm{~K})$, respectively. The real part of the FFCF as obtained from Eq. (43) is shown in Fig. 7. In this case, the CMD-based result is significantly different from, and presumably more accurate than, the harmonic-QCF-based result. The reason for this can be traced back to the fact that $C^{\mathrm{CMD}}(\tau)$ is significantly different from its classical counterpart in this more pronouncedly quantum mechanical liquid. The FT of the FFCF, as obtained from Eq. (44), is shown in Fig. 8 on a semilog plot. The high-frequency tail of Eq. (44) clearly deviate from that obtained via the harmonic QCF. Thus, in this case, the fact that we used CMD instead of classical MD does make a difference.

In order to get further insight into the above observations, it is instructive to consider an approximate version of CMD, which is based on centroid pseudo (pair) potentials (PP-CMD) ${ }^{83,94}$ instead of the full CMD potential (cf. Figs. 5, 6,7 , and 8). The centroid pseudopair potentials for the two cases considered above are shown in Fig. 9. In the case of neon, the CMD pair potential essentially coincides with the classical pair potential. This is not surprising since neon is a relatively heavy atom. This observation explains why using CMD instead of classical dynamics does not change the results in Figs. 5 and 6. At the same time, the smaller mass of

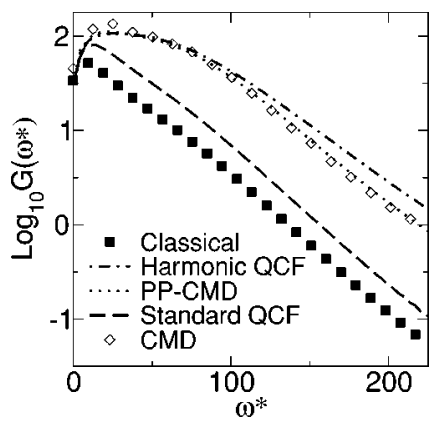

FIG. 8. A semilog plot of the FT of the FFCF for the breathing sphere helium model. Shown are results obtained from CMD, PP-CMD, classical mechanics, and the standard, harmonic, Schofield, and Egelstaff QCFs.

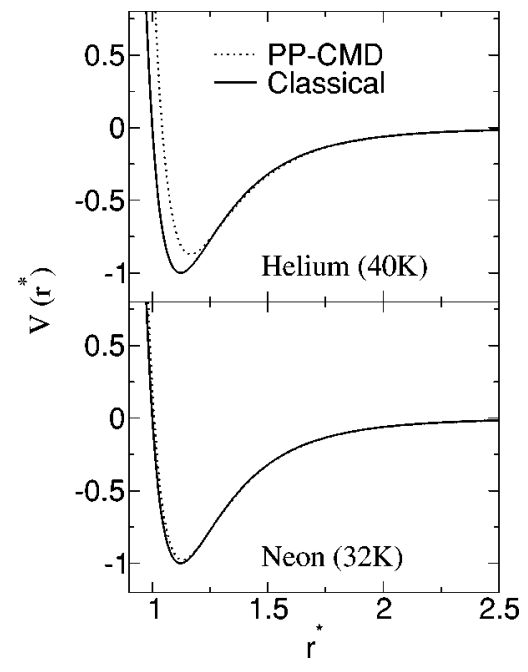

FIG. 9. Centroid pseudo pair potentials for the breathing sphere helium and neon models.

helium intensifies the quantum nature of the system, with the result that the centroid pseudopair potential becomes significantly different in comparison to the classical pair potential, which explains why using CMD does make a difference in this particular case.

\section{DISCUSSION}

VER of high-frequency molecular vibrations represents a rather unique example for an important, highly nonclassical, and inherently condensed-phase process. Experimental VER rate constants are often found to deviate from the corresponding classical predictions by several orders of magnitude, and bridging this gap represents an important test and a challenge for any method that aspires to account for quantum dynamical effects in condensed phase systems. It should be emphasized that in most cases of interest, classical mechanics would provide reasonable predictions of the bulk properties of the solvent, under the prevailing conditions. However, VER is extremely sensitive to these very few high-frequency, and therefore very quantum-mechanical, modes of the solvent that are in resonance with the high frequency of the relaxing vibrational mode. CMD has been observed in the past to be rather accurate in predicting such quantities as diffusion coefficients in pronouncedly quantum liquids. ${ }^{94}$ It has also been shown to work reasonably well for barrier crossing problems, where the extreme sensitivity of the rate to the barrier height seems to be in step with the idea of introducing quantum effects by means of an effective centroid potential. ${ }^{105,106}$ However, as we have shown in this paper, CMD can run into difficulties in the case of VER, where the centroid force is essentially dominated by the large majority of classical modes, which completely overwhelm, and obscure, the very few quantum modes that are relevant for VER. The fact that the VER rate constant is given in terms of a second order Kubo-transformed correlation function may have further contributed to the inaccuracy of CMD. For example, in the case of exponential coupling to a harmonic bath, CMD would give the exact first-order Kubotransformed correlation function, but not the second-order 
one. It should be noted however, that the rate constant for LT-type VER cannot be given in terms of a first-order Kubotransformed correlation function that can be directly obtained from CMD simulations (unless additional approximations are employed).

In retrospect, the above observations are perhaps not surprising if one takes into account the basic procedures used by CMD in order to account for quantum effects. Perhaps more surprising is the fact that the seemingly similar LSC-IVR method has been found to outperform CMD in the case of VER, and actually provides very accurate predictions of the VER rate constant. ${ }^{133}$ After all, LSC-IVR also leads to a classical-like expression for the FFCF, with non-classical sampling and "Wigner symbols," instead of centroid symbols, of the corresponding operators. In order to gain further insight into this observation, it is important to note that the high-frequency tail of the LSC-IVR FFCF power spectrum is dominated by a nonclassical term which is very sensitive to quantum fluctuations of the force around its average value. ${ }^{133}$ The centroid symbol of the force, Eq. (38), which correspond to the average force over the corresponding imaginary-time cyclic path, seem to miss this effect. Another evidence in favor of this interpretation comes from our recent work on the relationship between LSC-IVR and CMD. ${ }^{153}$ We found that the centroid correlation function can be obtained from the LSC-IVR correlation function, by decoupling the centroid, which corresponds to the zero-frequency normal mode of the corresponding imaginary-time cyclic path, from the higher normal modes. These higher normal modes, which LSC-IVR can account for, are responsible for the very same quantum fluctuations that seem to play a key role in VER. We note in passing that the analysis in Ref. 153 was performed in terms of first-order Kubo-transformed correlation functions, whereas our expression for the VER rate constant, Eq. (35), is given in terms of a second-order Kubotransformed correlation function. However, it seems reasonable to assume that the general argument remains valid for this case too.

The analysis presented in this paper is also valuable beyond the particular application to the VER problem. More specifically, we have shown that one can take advantage of the freedom in choosing the perturbation in linear-response theory, in order to relate rate constants to correlation functions that can be obtained directly from CMD simulations. It should be noted that the same idea has been recently employed for calculating reaction rate constants from CMD simulations. ${ }^{105,106}$ The general procedure, which avoids the need for adding approximations to CMD, should be applicable for any process that can be characterized by a rate constant. We hope that by utilizing this procedure one will be able to extend the application and testing of CMD to a wider range of problems than is currently available.

\section{ACKNOWLEDGMENTS}

The authors are grateful for financial support from the National Science Foundation through Grant No. CHE0306695 .

\section{APPENDIX A: THE LANDAU-TELLER LIMIT}

In this appendix, we outline the derivation of Eq. (18) from Eq. (34) in the LT limit. To this end, one has to find the leading terms in the expansion of $k$, Eq. (34), in powers of $\alpha$. It can be readily shown that the leading term in the expansion of the denominator is of 0th order in $\alpha$, while that in the expansion of the numerator is second order in $\alpha$. Thus, the leading term in the expansion of $k$ is also of second order in $\alpha$, and the corresponding coefficient is given by

$$
\begin{aligned}
k^{(2)}= & \frac{2 \omega^{2}}{Z_{s}^{0} Z_{b}^{0}}\left[\int _ { 0 } ^ { \beta } d \beta _ { 1 } \int _ { 0 } ^ { \beta _ { 1 } } d \beta _ { 2 } \operatorname { T r } \left\{e^{-\beta \hat{H}} \delta \hat{q}\left(-i \beta_{1} / \hbar\right)\right.\right. \\
& \left.\left.\times \delta \hat{q}\left(-i \beta_{2} / \hbar\right)[\hat{p} \hat{F}](t)\right\}\right]_{1} .
\end{aligned}
$$

Here $Z_{s}^{0}=\operatorname{Tr}_{s}\left(e^{-\beta \hat{H}_{s}}\right), Z_{b}^{0}=\operatorname{Tr}_{b}\left(e^{-\beta \hat{H}_{b}}\right)$, and $[\cdots]_{1}$ is the first-order term, with respect to $\alpha$, of the corresponding expression.

It should be noted that $e^{-\beta \hat{H}}, \quad \delta \hat{q}\left(-i \hbar \beta_{1}\right), \quad \delta \hat{q}$ $\left(-i \hbar \beta_{2}\right)$, and $[\hat{p} \hat{F}](t)$ all depend explicitly on $\alpha$, and have the following nonvanishing zero and first-order terms when expanded in powers of $\alpha$ :

$$
\begin{aligned}
& {\left[e^{-\beta \hat{H}}\right]_{0}=e^{-\beta \hat{H}_{0},}} \\
& {\left[e^{-\beta \hat{H}}\right]_{1}=-e^{-\beta \hat{H}_{0}} \int_{0}^{\beta} d \beta_{1} \hat{q}_{0}\left(-i \hbar \beta_{1}\right) \hat{F}_{0}\left(-i \hbar \beta_{1}\right),} \\
& {\left[\delta \hat{q}\left(-i \hbar \beta_{1}\right)\right]_{0}=\hat{q}_{0}\left(-i \hbar \beta_{1}\right),} \\
& {\left[\delta \hat{q}\left(-i \hbar \beta_{1}\right)\right]_{1}=\frac{\hbar}{2 \mu \omega} \int_{0}^{\beta_{1}} d \lambda \hat{F}_{0}(-i \hbar \lambda)\left[e^{\left(\beta_{1}-\lambda\right) \hbar \omega}\right.} \\
& {[[\hat{p} \hat{F}](t)]_{0}=\hat{p}_{0}(t) \hat{F}_{0}(t),} \\
& {[[\hat{p} \hat{F}](t)]_{1}=\frac{i}{\hbar} \int_{0}^{t} d t_{1}\left[\hat{q}_{0}\left(t_{1}\right) \hat{p}_{0}(t) \hat{F}_{0}\left(t_{1}\right) \hat{F}_{0}(t)\right.} \\
& \left.-\hat{p}_{0}(t) \hat{q}_{0}\left(t_{1}\right) \hat{F}_{0}(t) \hat{F}_{0}\left(t_{1}\right)\right] .
\end{aligned}
$$

Here $\langle\hat{A}\rangle_{0}=\operatorname{Tr}\left(\hat{A} e^{-\beta \hat{H}_{0}}\right) / Z^{0}$ and $\hat{A}_{0}(\tau)=e^{i \hat{H}_{0} \tau / \hbar} \hat{A} e^{-i \hat{H}_{0} \tau / \hbar}$.

Equation (A1) can then be written more explicitly as follows: 


$$
\begin{aligned}
k^{(2)}= & \frac{2 \omega^{2}}{Z_{s}^{0} Z_{b}^{0}}\left[\int_{0}^{\beta} d \beta_{1} \int_{0}^{\beta_{1}} d \beta_{2} \operatorname{Tr}\left\{\left[e^{-\beta \hat{H}}\right]_{1} \hat{q}_{0}\left(-i \beta_{1} / \hbar\right) \hat{q}_{0}\left(-i \beta_{2} / \hbar\right) \hat{p}_{0}(t) \hat{F}_{0}(t)\right\}\right. \\
& +\int_{0}^{\beta} d \beta_{1} \int_{0}^{\beta_{1}} d \beta_{2} \operatorname{Tr}\left\{e^{-\beta \hat{H}_{0}}\left[\delta \hat{q}\left(-i \beta_{1} / \hbar\right)\right]_{1} \hat{q}_{0}\left(-i \beta_{2} / \hbar\right) \hat{p}_{0}(t) \hat{F}_{0}(t)\right\}+\int_{0}^{\beta} d \beta_{1} \int_{0}^{\beta_{1}} d \beta_{2} \operatorname{Tr}\left\{e^{-\beta \hat{H}_{0}} \hat{q}_{0}\left(-i \beta_{1} / \hbar\right)\right. \\
& \left.\left.\times\left[\delta \hat{q}\left(-i \beta_{2} / \hbar\right)\right]_{1} \hat{p}_{0}(t) \hat{F}_{0}(t)\right\}+\int_{0}^{\beta} d \beta_{1} \int_{0}^{\beta_{1}} d \beta_{2} \operatorname{Tr}\left\{e^{-\beta \hat{H}_{0}} \hat{q}_{0}\left(-i \beta_{1} / \hbar\right) \hat{q}_{0}\left(-i \beta_{2} / \hbar\right)[[\hat{p} \hat{F}](t)]_{1}\right\}\right] .
\end{aligned}
$$

In order to proceed, one has to substitute the corresponding zeroth and first-order terms from Eqs. (A2) into (A3). The resulting equation can then be further simplified to yield

$$
k^{(2)}(t)=[\mathcal{L}(t)+\mathcal{R}(t)+\mathcal{T}(t)]
$$

where

$$
\begin{aligned}
\mathcal{L}(t)= & \frac{1}{\mu \hbar \omega} \operatorname{Re}\left\{\left(1+\frac{\beta \hbar \omega}{e^{\beta \hbar \omega}-1}\right) \int_{0}^{t} d \tau e^{i \omega \tau} C(\tau)\right. \\
& \left.-\left(1+\frac{\beta \hbar \omega}{1-e^{-\beta \hbar \omega}}\right) \int_{0}^{t} d \tau e^{-i \omega \tau} C(\tau)\right\}, \\
\mathcal{R}(t)= & \frac{1}{\mu \hbar \omega} \operatorname{Re}\left\{e^{2 i \omega t} \int_{0}^{t} d \tau e^{i \omega \tau}[C(\tau)-C(-\tau)]\right\},
\end{aligned}
$$

and

$$
\begin{aligned}
\mathcal{T}(t)= & \frac{1}{\mu \omega} \operatorname{Re}\left\{\frac { \beta \hbar \omega } { 1 - e ^ { - \beta \hbar \omega } } \operatorname { I m } \left[e^{i \omega t}\right.\right. \\
& \left.\times \int_{0}^{\beta} d \lambda e^{-\lambda \hbar \omega} C(-t-i \hbar \lambda)\right] \\
& \left.+2 \sin (\omega t) \int_{0}^{\beta} d \lambda C(-t-i \hbar \lambda)\right\} .
\end{aligned}
$$

The LT formula emerges from Eq. (A4) when the two additional approximations of BRT are imposed on it:

(1) Within BRT, rate kinetics is obtained at the Markovian limit, which is reached when $t$ becomes larger than the correlation time of the FFCF, denoted by $\tau_{c} . \tau_{c}$ is typically very short in liquids $(\sim \mathrm{ps})$, particularly in comparison to the VER lifetime, $T_{1}$. In the case of Eq. (34), $\tau_{c}$ corresponds to the plateau time that signals the onset of rate kinetics. Assuming that $t>\tau_{c}$ in Eq. (A4) then has two implications: (1) The transient term, $\mathcal{T}(t)$, vanishes $\left[C(-t-i \hbar \lambda) \rightarrow 0\right.$ at $\left.t>\tau_{c}\right]$; (2) the limit of the time integrals in the terms $\mathcal{L}(t)$ and $\mathcal{R}(t)$ can be extended to $\infty$.

(2) Decoupling the population and phase relaxations within BRT also requires the averaging out of terms which rotate rapidly with the frequency $2 \omega$ (the RWA). Applying this approximation to Eq. (A4) leads to the removal of the rotating term $\mathcal{R}(t)$.
Thus, under these two approximations, $k^{(2)}$ reduces into $\lim _{t \rightarrow \infty} \mathcal{L}(t)$. It is then easy to show, with the help of the following identities:

$$
\int_{0}^{\infty} d \tau e^{i \omega \tau} C(\tau)=\frac{1}{2} \widetilde{C}(\omega)+\frac{1}{2 \pi i} \mathcal{P} \int_{-\infty}^{\infty} d \omega^{\prime} \frac{\widetilde{C}\left(\omega^{\prime}\right)}{\omega^{\prime}-\omega},
$$

$$
\widetilde{C}(-\omega)=e^{-\beta \hbar \omega} \widetilde{C}(\omega),
$$

that

$$
k^{(2)} \approx \lim _{t \rightarrow \infty} \mathcal{L}(t)=\frac{1-e^{-\beta \hbar \omega}}{\beta \hbar \omega} \frac{\beta}{2 \mu} \widetilde{C}(\omega),
$$

which is identical to $1 / T_{1}$ in the LT formula, Eq. (18).

\section{APPENDIX B: THE DERIVATION OF THE CENTROID LANDAU-TELLER FORMULA}

In this Appendix, we outline the derivation of the centroid Landau-Teller formula, Eq. (41), from Eq. (35). To this end, one has to find the leading terms in the expansion of $k_{\mathrm{CMD}}$, Eq. (35), in powers of $\alpha$. The leading term in the expansion of the denominator is of 0th order in $\alpha$, and is given by

$$
\begin{aligned}
& {\left[\int d q _ { c } \int d p _ { c } \int d \mathbf { Q } _ { \mathbf { c } } \int d \mathbf { P } _ { \mathbf { c } } \operatorname { e x p } \left\{-\beta\left[\sum_{i=1}^{N} \frac{\left(P_{c}^{(i)}\right)^{2}}{2 M^{(i)}}\right.\right.\right.} \\
& \left.\left.\left.+\frac{p_{c}^{2}}{2 m}+U_{c m}\left(\mathbf{Q}_{\mathbf{c}}, q_{c}\right)\right]\right\}\left[\delta q_{c}\right]^{2}\left[\delta H_{s}\right]_{c}\left(q_{c}, p_{c}, \mathbf{Q}_{\mathbf{c}}, \mathbf{P}_{\mathbf{c}}\right)\right]_{0} \\
& =Z_{b}^{0} \frac{2 \pi}{\mu \beta^{3} \omega^{3}} \frac{\beta \hbar \omega / 2}{\sinh (\beta \hbar \omega / 2)},
\end{aligned}
$$

where we have used Eqs. (55) and (72) of Ref. 89 for the centroid density and energy centroid symbol of a free harmonic oscillator, respectively. The first nonvanishing term in the expansion of the numerator is second order in $\alpha$. It should be noted that $\rho_{c}\left(\mathbf{Q}_{\mathbf{c}}, q_{c}\right), \delta q_{c}(-t)$, and $F_{c}\left(\mathbf{Q}_{\mathbf{c}}, q_{c}\right)$ all depend explicitly on $\alpha$. The corresponding zero and first order terms obtained when these quantities are expanded in powers of $\alpha$ are as follows:

$$
\begin{aligned}
& {\left[\rho_{c}\left(\mathbf{Q}_{\mathbf{c}}, q_{c}\right)\right]_{0}=\rho_{c}^{s}\left(q_{c}\right) \rho_{c}^{b}\left(\mathbf{Q}_{\mathbf{c}}\right),} \\
& {\left[\rho_{c}\left(\mathbf{Q}_{\mathbf{c}}, q_{c}\right)\right]_{1}=-\beta \rho_{c}^{s}\left(q_{c}\right) \rho_{c}^{b}\left(\mathbf{Q}_{\mathbf{c}}\right) q_{c} F_{c}\left(\mathbf{Q}_{\mathbf{c}}\right),}
\end{aligned}
$$




$$
\begin{aligned}
{\left[\delta q_{c}(-t)\right]_{0}=} & q_{c} \cos (\omega t)-\frac{p_{c}}{\mu \omega} \sin (\omega t), \\
{\left[\delta q_{c}(-t)\right]_{1}=} & -\frac{1}{\mu \omega} \int_{0}^{t} d \tau \sin [\omega(t-\tau)] F_{c}\left[\mathbf{Q}_{\mathbf{c}}(-\tau)\right] \\
& +\frac{1}{\mu \omega^{2}}\left\langle F_{c}\left(\mathbf{Q}_{\mathbf{c}}\right)\right\rangle_{c}, \\
{\left[F_{c}\left(\mathbf{Q}_{\mathbf{c}}, q_{c}\right)\right]_{0}=} & F_{c}\left(\mathbf{Q}_{\mathbf{c}}\right),\left[F_{c}\left(\mathbf{Q}_{\mathbf{c}}, q_{c}\right)\right]_{1}=0 .
\end{aligned}
$$

Using the zeroth and first order terms from Eqs. (B2) in order to find the leading second order term in the expansion of the denominator of Eq. (35), followed by some algebra, then leads to the following result:

$$
k_{\mathrm{CMD}}^{(2)}=\left[\mathcal{L}_{\mathrm{CMD}}(t)+\mathcal{R}_{\mathrm{CMD}}(t)\right],
$$

where

$$
\mathcal{L}_{\mathrm{CMD}}(t)=\frac{\beta}{\mu} \int_{0}^{t} d \tau \cos (\omega \tau) C^{\mathrm{CMD}}(\tau),
$$

and

$$
\begin{aligned}
F_{c}\left(\mathbf{Q}_{\mathbf{c}}\right)= & \prod_{j=1}^{N}\left\{\int d Q_{1}^{(j)} \cdots \int d Q_{P}^{(j)} e^{c^{(j)} \sqrt{2 M^{(j)} \omega^{(j)} / \hbar} Q_{1}^{(j)}} \delta\left(Q_{c}^{(j)}-P^{-1} \sum_{k=1}^{P} Q_{k}^{(j)}\right) e^{-\beta \Sigma_{k=1}^{P}\left[1 / 2 M^{(j)} \omega_{P}^{2}+(1 / P) 1 / 2 M^{(j)}\left(\omega^{(j)}\right)^{2}\right]\left(Q_{k}^{(j)}\right)^{2}}\right\} \\
& \left.\times\left\{\int d Q_{1}^{(j)} \cdots \int d Q_{P}^{(j)} \delta\left(Q_{c}^{(j)}-P^{-1} \sum_{k=1}^{P} Q_{k}^{(j)}\right) e^{-\beta \Sigma_{k=1}^{P}\left[1 / 2 M^{(j)} \omega_{P}^{2}+(1 / P) 1 / 2 M^{(j)}\left(\omega^{(j)}\right)^{2}\left(Q_{k}^{(j)}\right)^{2}\right]}\right\} \cdot\right\}^{-1} \cdot(\mathrm{C} 1)
\end{aligned}
$$

$$
\begin{aligned}
\mathcal{R}_{\mathrm{CMD}}(t)= & \frac{\beta}{\mu}\left\{\frac{\sin (2 \omega t)}{\omega} C^{\mathrm{CMD}}(0)\right. \\
& -\cos (2 \omega t) \int_{0}^{t} d \tau \cos (\omega \tau) C^{\mathrm{CMD}}(\tau) \\
& \left.-\sin (2 \omega t) \int_{0}^{t} d \tau \sin (\omega \tau) C^{\mathrm{CMD}}(\tau)\right\} .
\end{aligned}
$$

The centroid LT formula emerges from Eq. (B3) when Thus, assuming that $t>\tau_{c}$, where $\tau_{c}$ is the correlation time of $C^{\mathrm{CMD}}(\tau)$, implies that we can extend the limit of the time integrals in Eqs. (B4) and (B5) to $\infty$. Averaging out of terms which rotate rapidly with the frequency $2 \omega$ (the RWA) then leads to the removal of the rotating term $\mathcal{R}_{\mathrm{CMD}}(t) . k_{\mathrm{CMD}}^{(2)}$ reduces as a result into $\lim _{t \rightarrow \infty} \mathcal{L}_{\mathrm{CMD}}(t)$, which coincides with Eq. (41).

\section{APPENDIX C: EXPONENTIAL COUPLING TO THE HARMONIC BATH: THE CENTROID FORCE}

In this appendix, we outline the derivation of Eq. (52). To this end, we consider the centroid symbol of the exponential force operator, Eq. (46), the two additional approximations of BRT are imposed on it.
Changing the integration variables from $\left\{Q_{1}^{(j)} \cdots Q_{P}^{(j)}\right\}$ into the normal mode coordinates, ${ }^{105}\left\{\widetilde{Q}_{1}^{(j)} \cdots \widetilde{Q}_{P}^{(j)}\right\}$, noting that $\widetilde{Q}_{1}^{(j)}=P^{-1} \sum_{k=1}^{P} Q_{k}^{(j)}$ corresponds to the centroid, and explicitly integrating over the other normal modes, then leads to the following conclusion:

$$
F_{c}\left(\mathbf{Q}_{\mathbf{c}}\right)=\zeta e^{\Sigma_{j=1}^{N} c^{(j)} \sqrt{2 M^{(j)} \omega^{(j)} / \hbar} Q_{c}^{(j)}} .
$$

The value of $\zeta$ is determined by noting that

$$
\langle F(\hat{\mathbf{Q}})\rangle_{\mathrm{eq}}=\frac{\int d \mathbf{Q}_{\mathrm{c}} \rho_{c}^{b}\left(\mathbf{Q}_{\mathbf{c}}\right) F_{c}\left(\mathbf{Q}_{\mathbf{c}}\right)}{\int d \mathbf{Q}_{\mathbf{c}} \rho_{c}^{b}\left(\mathbf{Q}_{\mathbf{c}}\right)}
$$

and

$$
\langle F(\hat{\mathbf{Q}})\rangle_{\mathrm{Cl}}=\frac{\int d \mathbf{Q}_{\mathbf{c}} \rho_{c}^{b}\left(\mathbf{Q}_{\mathbf{c}}\right) F\left(\mathbf{Q}_{\mathbf{c}}\right)}{\int d \mathbf{Q}_{\mathbf{c}} \rho_{c}^{b}\left(\mathbf{Q}_{\mathbf{c}}\right)},
$$

which leads to Eq. (53).

\footnotetext{
${ }^{1}$ D. W. Oxtoby, Adv. Chem. Phys. 47, (Part 2), 487 (1981).

${ }^{2}$ D. W. Oxtoby, Annu. Rev. Phys. Chem. 32, 77 (1981).

${ }^{3}$ D. W. Oxtoby, J. Phys. Chem. 87, 3028 (1983).

${ }^{4}$ J. Chesnoy and G. M. Gale, Ann. Phys. (Paris) 9, 893 (1984).

${ }_{5}^{5}$ J. Chesnoy and G. M. Gale, Adv. Chem. Phys. 70, (Part 2), 297 (1988).

${ }^{6}$ C. B. Harris, D. E. Smith, and D. J. Russell, Chem. Rev. 90, 481 (1990).

${ }^{7}$ D. W. Miller and S. A. Adelman, Int. Rev. Phys. Chem. 13, 359 (1994).

${ }^{8}$ R. M. Stratt and M. Maroncelli, J. Phys. Chem. 100, 12981 (1996).
} 
${ }^{30}$ A. Salloum and H. Dubost, Chem. Phys. 189, 179 (1994).

${ }^{31}$ A. Tokmakoff, B. Sauter, and M. D. Fayer, J. Chem. Phys. 100, 9035 (1994).

${ }^{32}$ A. Tokmakoff and M. D. Fayer, J. Chem. Phys. 103, 2810 (1995).

${ }^{33}$ R. S. Urdahl et al., J. Chem. Phys. 107, 3747 (1997).

${ }^{34}$ J. C. Owrutsky, M. Li, B. Locke, and R. M. Hochstrasser, J. Phys. Chem. 99, 4842 (1995).

${ }^{35}$ R. Laenen, C. Rauscher, and A. Laubereau, Phys. Rev. Lett. 80, 2622 (1998).

${ }^{36}$ S. Woutersen, U. Emmerichs, H. Nienhuys, and H. J. Bakker, Phys. Rev. Lett. 81, 1106 (1998).

${ }^{37}$ D. J. Myers et al., J. Chem. Phys. 109, 5971 (1998).

${ }^{38}$ D. E. Sagnella et al., Proc. Natl. Acad. Sci. U.S.A. 96, 14324 (1999).

${ }^{39}$ P. Hamm, M. Lim, and R. M. Hochstrasser, J. Chem. Phys. 107, 1523 (1997).

${ }^{40}$ Q. Shi and E. Geva, J. Chem. Phys. 118, 7562 (2003).

${ }^{41}$ A. Nitzan, S. Mukamel, and J. Jortner, J. Chem. Phys. 60, 3929 (1974).

${ }^{42}$ A. Nitzan, S. Mukamel, and J. Jortner, J. Chem. Phys. 63, 200 (1975).

${ }^{43}$ S. A. Egorov and J. L. Skinner, J. Chem. Phys. 105, 7047 (1996).

${ }^{44}$ K. F. Everitt, S. A. Egorov, and J. L. Skinner, Chem. Phys. 235, 115 (1998).

${ }^{45}$ K. F. Everitt and J. L. Skinner, J. Chem. Phys. 110, 4467 (1999).

${ }^{46}$ J. Poulsen and P. J. Rossky, J. Chem. Phys. 115, 8014 (2001).

${ }^{47}$ D. C. Douglass, J. Chem. Phys. 35, 81 (1961).

${ }^{48}$ S. A. Adelman and R. H. Stote, J. Chem. Phys. 88, 4397 (1988).

${ }^{49}$ R. H. Stote and S. A. Adelman, J. Chem. Phys. 88, 4415 (1988).

${ }^{50}$ S. A. Adelman, R. Muralidhar, and R. H. Stote, J. Chem. Phys. 95, 2738 (1991).

${ }^{51}$ E. Rabani and D. R. Reichman, J. Phys. Chem. B 105, 6550 (2001).

${ }^{52}$ N. Makri, Annu. Rev. Phys. Chem. 50, 167 (1999).

${ }^{53}$ B. J. Berne, J. Jortner, and R. Gordon, J. Chem. Phys. 47, 1600 (1967).

${ }^{54}$ J. S. Bader and B. J. Berne, J. Chem. Phys. 100, 8359 (1994).

${ }^{55}$ S. A. Egorov, K. F. Everitt, and J. L. Skinner, J. Phys. Chem. A 103, 9494 (1999).

${ }^{56}$ S. A. Egorov and J. L. Skinner, J. Chem. Phys. 112, 275 (2000).

${ }^{57}$ J. L. Skinner and K. Park, J. Phys. Chem. B 105, 6716 (2001).

${ }^{58}$ D. Rostkier-Edelstein, P. Graf, and A. Nitzan, J. Chem. Phys. 107, 10470 (1997).

${ }^{59}$ D. Rostkier-Edelstein, P. Graf, and A. Nitzan, J. Chem. Phys. 108, 9598 (1998).

${ }^{60}$ K. F. Everitt, J. L. Skinner, and B. M. Ladanyi, J. Chem. Phys. 116, 179 (2002).

${ }^{61}$ P. H. Berens, S. R. White, and K. R. Wilson, J. Chem. Phys. 75, 515 (1981).

${ }^{62}$ L. Frommhold, in Cambridge Monographs on Atomic, Molecular, and Chemical Physics, 1st ed. (Cambridge University Press, England, 1993), Vol. 2.

${ }^{63}$ J. L. Skinner, J. Chem. Phys. 107, 8717 (1997).

${ }^{64}$ S. C. An, C. J. Montrose, and T. A. Litovitz, J. Chem. Phys. 64, 3717 (1976).

${ }^{65}$ S. A. Egorov and J. L. Skinner, Chem. Phys. Lett. 293, 439 (1998).

${ }^{66}$ P. Schofield, Phys. Rev. Lett. 4, 239 (1960).

${ }^{67}$ P. A. Egelstaff, Adv. Phys. 11, 203 (1962).

${ }^{68}$ G. R. Kneller, Mol. Phys. 83, 63 (1994).

${ }^{69}$ G. D. Billing, Chem. Phys. Lett. 30, 391 (1975).

${ }^{70}$ G. D. Billing, J. Chem. Phys. 99, 5849 (1993).

${ }^{71}$ J. C. Tully and R. K. Preston, J. Chem. Phys. 55, 562 (1971).

${ }^{72}$ J. C. Tully, J. Chem. Phys. 93, 1061 (1990).

${ }^{73}$ P. J. Kuntz, J. Chem. Phys. 95, 141 (1991).

${ }^{74}$ A. I. Krylov et al., J. Chem. Phys. 104, 3651 (1996).

${ }^{75}$ K. Yamashita and W. H. Miller, J. Chem. Phys. 82, 5475 (1985).

${ }^{76}$ E. Gallicchio and B. J. Berne, J. Chem. Phys. 105, 7064 (1996).

${ }^{77}$ E. Gallicchio, S. A. Egorov, and B. J. Berne, J. Chem. Phys. 109, 7745 (1998).

${ }^{78}$ S. A. Egorov, E. Gallicchio, and B. J. Berne, J. Chem. Phys. 107, 9312 (1997).

${ }^{79}$ G. Krilov and B. J. Berne, J. Chem. Phys. 111, 9147 (1999).

${ }^{80}$ E. Rabani, G. Krilov, and B. J. Berne, J. Chem. Phys. 112, 2605 (2000).

${ }^{81}$ E. Sim, G. Krilov, and B. Berne, J. Phys. Chem. A 105, 2824 (2001).

${ }^{82}$ S. Jang, Y. Pak, and G. A. Voth, J. Phys. Chem. A 103, 10289 (1999).

${ }^{83}$ J. Cao and G. A. Voth, J. Chem. Phys. 100, 5093 (1994).

${ }^{84}$ J. Cao and G. A. Voth, J. Chem. Phys. 100, 5106 (1994).

${ }^{85}$ J. Cao and G. A. Voth, J. Chem. Phys. 101, 6157 (1994).
${ }^{86}$ J. Cao and G. A. Voth, J. Chem. Phys. 101, 6168 (1994).

${ }^{87}$ J. Cao and G. A. Voth, J. Chem. Phys. 101, 6184 (1994).

${ }^{88}$ G. A. Voth, Adv. Chem. Phys. 93, 135 (1996).

${ }^{89}$ S. Jang and G. A. Voth, J. Chem. Phys. 111, 2357 (1999).

${ }^{90}$ S. Jang and G. A. Voth, J. Chem. Phys. 111, 2371 (1999).

${ }^{91}$ D. R. Reichman, P.-N. Roy, S. Jang, and G. A. Voth, J. Chem. Phys. 113, 919 (2000).

${ }^{92}$ A. Calhoun, M. Pavese, and G. A. Voth, Chem. Phys. Lett. 262, 415 (1996).

${ }^{93}$ U. W. Schmitt and G. A. Voth, J. Chem. Phys. 111, 9361 (1999).

${ }^{94}$ M. Pavese and G. A. Voth, Chem. Phys. Lett. 249, 231 (1996).

${ }^{95}$ K. Kinugawa, P. B. Moore, and M. L. Klein, J. Chem. Phys. 106, 1154 (1997).

${ }^{96}$ K. Kinugawa, P. B. Moore, and M. L. Klein, J. Chem. Phys. 109, 610 (1998).

${ }^{97}$ K. Kinugawa, Chem. Phys. Lett. 292, 454 (1998).

${ }^{98}$ M. Pavese, D. R. Bernard, and G. A. Voth, Chem. Phys. Lett. 300, 93 (1999).

${ }^{99}$ R. Ramirez, T. Lopez-Ciudad, and J. C. Noya, Phys. Rev. Lett. 81, 3303 (1998).

${ }^{100}$ R. Ramirez and T. Lopez-Ciudad, Phys. Rev. Lett. 83, 4456 (1999).

${ }^{101}$ R. Ramirez and T. Lopez-Ciudad, J. Chem. Phys. 111, 3339 (1999).

${ }^{102}$ T. Lopez-Ciudad and R. Ramirez, J. Chem. Phys. 113, 10849 (2000).

${ }^{103}$ J. Poulsen, S. R. Keiding, and P. J. Rossky, Chem. Phys. Lett. 336, 488 (2001).

${ }^{104}$ J. Poulsen and P. J. Rossky, J. Chem. Phys. 115, 8024 (2001).

${ }^{105}$ E. Geva, Q. Shi, and G. A. Voth, J. Chem. Phys. 115, 9209 (2001).

${ }^{106}$ Q. Shi and E. Geva, J. Chem. Phys. 116, 3223 (2002).

${ }^{107}$ E. Rabani and D. R. Reichman, J. Chem. Phys. 116, 6271 (2002).

${ }^{108}$ E. Rabani and D. R. Reichman, Phys. Rev. E 65, 036111 (2002).

${ }^{109}$ D. R. Reichman and E. Rabani, J. Chem. Phys. 116, 6279 (2002).

${ }^{110}$ H. Wang, X. Sun, and W. H. Miller, J. Chem. Phys. 108, 9726 (1998).

${ }^{111}$ E. Pollak and J. Liao, J. Chem. Phys. 108, 2733 (1998).

${ }^{112}$ W. H. Miller, Adv. Chem. Phys. 25, 69 (1974).

${ }^{113}$ W. H. Miller, J. Chem. Phys. 53, 3578 (1970).

${ }^{114}$ M. F. Herman and E. Kluk, Chem. Phys. 91, 27 (1984).

${ }^{115}$ E. J. Heller, J. Chem. Phys. 94, 2723 (1981).

${ }^{116}$ K. G. Kay, J. Chem. Phys. 100, 4377 (1994).

${ }^{117}$ M. Ovchinnikov and V. A. Apkarian, J. Chem. Phys. 105, 10312 (1996).

${ }^{118}$ M. Ovchinnikov and V. A. Apkarian, J. Chem. Phys. 108, 2277 (1998).

${ }^{119}$ X. Sun and W. H. Miller, J. Chem. Phys. 106, 916 (1997).

${ }^{120}$ N. Makri and K. Thompson, Chem. Phys. Lett. 291, 101 (1998).

${ }^{121}$ W. H. Miller, Faraday Discuss. 110, 1 (1998).

${ }^{122}$ J. S. Shao and N. Makri, J. Phys. Chem. A 103, 7753 (1999).

${ }^{123}$ K. Thompson and N. Makri, Phys. Rev. E 59, R4729 (1999).

${ }^{124}$ O. Kühn and N. Makri, J. Phys. Chem. A 103, 9487 (1999).

${ }^{125}$ H. Wang, M. Thoss, and W. H. Miller, J. Chem. Phys. 112, 47 (2000).

${ }^{126}$ M. Ovchinnikov, V. A. Apkarian, and G. A. Voth, J. Chem. Phys. 184, 7130 (2001).

${ }^{127}$ W. H. Miller, J. Phys. Chem. A 105, 2942 (2001).

${ }^{128}$ N. Makri and W. H. Miller, J. Chem. Phys. 116, 9207 (2002).

${ }^{129}$ X. Sun, H. Wang, and W. H. Miller, J. Chem. Phys. 109, 4190 (1998).

${ }^{130}$ X. Sun, H. Wang, and W. H. Miller, J. Chem. Phys. 109, 7064 (1998).

${ }^{131}$ H. Wang, X. Song, D. Chandler, and W. H. Miller, J. Chem. Phys. 110, 4828 (1999).

${ }^{132}$ X. Sun and W. H. Miller, J. Chem. Phys. 110, 6635 (1999).

${ }^{133}$ Q. Shi and E. Geva, J. Phys. Chem. A (in press).

${ }^{134}$ B. J. Berne and D. Thirumalai, Annu. Rev. Phys. Chem. 37, 401 (1986).

${ }^{135}$ D. M. Ceperley, Rev. Mod. Phys. 67, 279 (1995).

${ }^{136}$ A. Nitzan and R. Silbey, J. Chem. Phys. 60, 4070 (1974).

${ }^{137}$ F. E. Figueirido and R. M. Levy, J. Chem. Phys. 97, 703 (1992).

${ }^{138}$ A. G. Redfield, IBM Syst. J. 1, 19 (1957).

${ }^{139}$ R. K. Wangsness and F. Bloch, Phys. Rev. 89, 728 (1953).

${ }^{140}$ C. P. Slichter, Principles of Magnetic Resonance (Springer-Verlag, Berlin, 1990).

${ }^{141}$ A. Abragam, The Principles of Nuclear Magnetism (Oxford, London, 1961).

${ }^{142}$ J. M. Jean, R. A. Friesner, and G. R. Fleming, J. Chem. Phys. 96, 5827 (1992).

${ }^{143}$ H. Gai and G. A. Voth, J. Chem. Phys. 99, 740 (1993). 
${ }^{144}$ W. T. Pollard and R. A. Friesner, J. Chem. Phys. 100, 5054 (1994).

${ }^{145}$ E. Geva, E. Rosenman, and D. J. Tannor, J. Chem. Phys. 113, 1380 (2000).

${ }^{146}$ N. G. V. Kampen, Stochastic Processes in Physics and Chemistry (Elsevier, Amsterdam, 1992).

${ }^{147}$ R. Zwanzig, J. Chem. Phys. 34, 1931 (1961).

${ }^{148}$ L. Landau and E. Teller, Physik Z. Sobyetunion 10, 34 (1936).
${ }^{149}$ R. Kubo, M. Toda, and N. Hashitsume, Statistical Physics II: Nonequilibrium Statistical Mechanics (Springer-Verlag, Berlin, 1983).

${ }^{150}$ J. Chesnoy and J. J. Weis, J. Chem. Phys. 84, 5378 (1986).

${ }^{151}$ J. Hautman and M. L. Klein, Mol. Phys. 80, 647 (1993).

${ }^{152}$ J. A. Barker, D. Henderson, and F. F. Abraham, Physica A 106, 226 (1981).

${ }^{153}$ Q. Shi and E. Geva, J. Chem. Phys. 118, 8173 (2003). 